SINAI Journal of Applied Sciences (ISSN: 2314-6079), Vol. (8), Is. (1), Apr. 2019

\begin{tabular}{|c|c|}
\hline 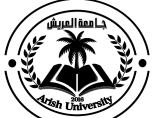 & $\begin{array}{l}\text { SCREENED By SINAI Journal of Applied Sciences } \\
\text { iThenticate }\end{array}$ \\
\hline
\end{tabular}

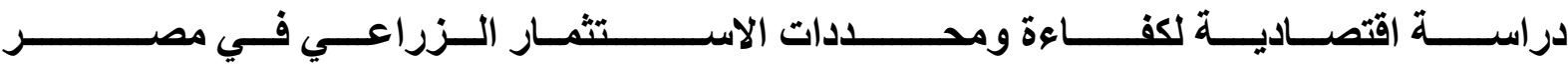
وسام إيهاب محمد إبراهيم الأثرم"، رياض إسماعيل مصطفي رضوان، سعاد عبدالفتاح إبراهيم، رجب محمد حفني

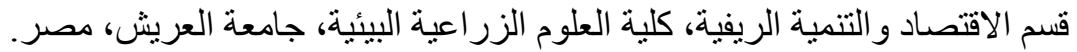

\title{
الملخص
}

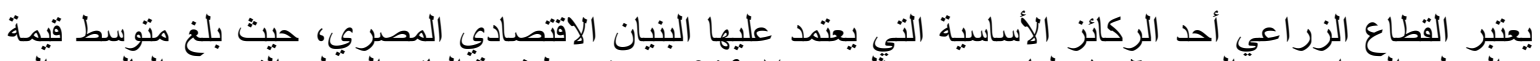

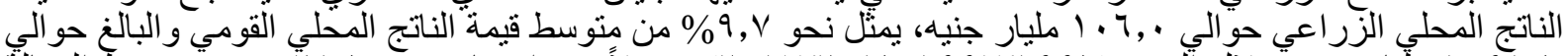

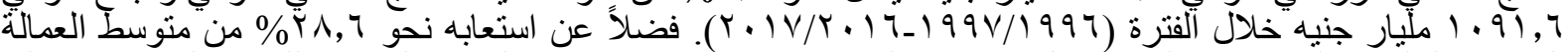

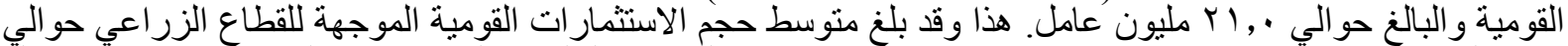

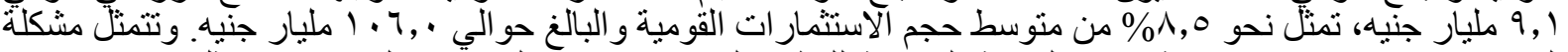

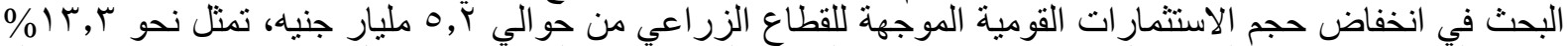

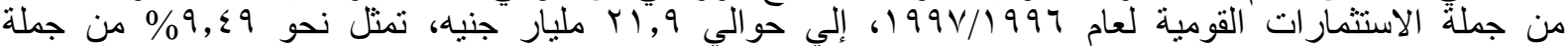

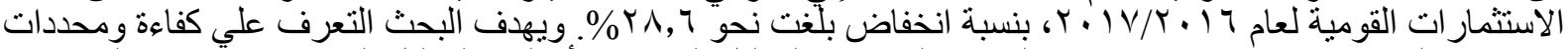

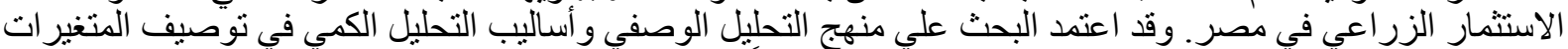

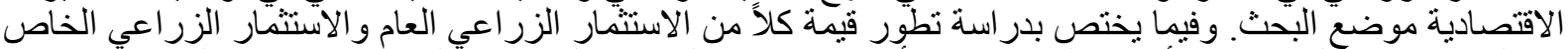

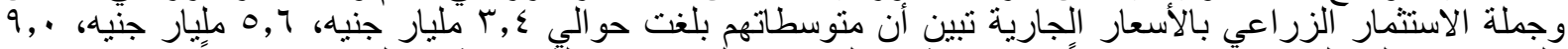

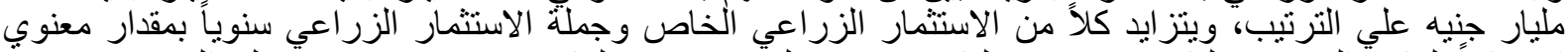

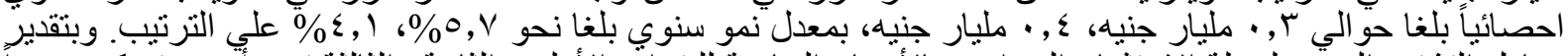

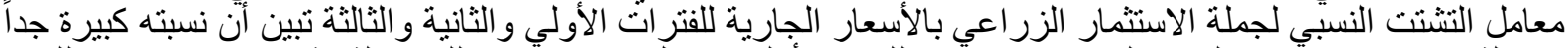

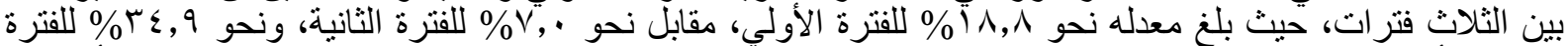

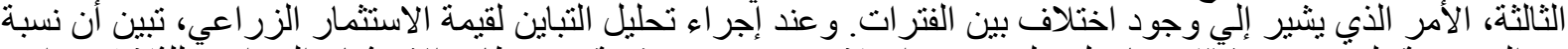

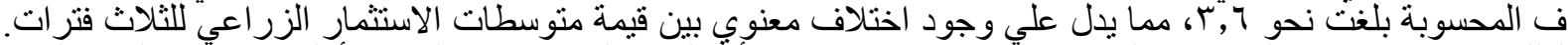

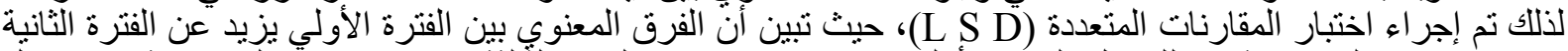

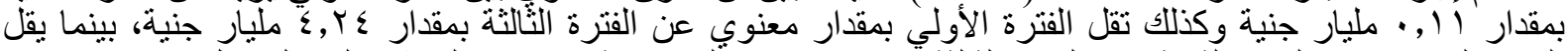

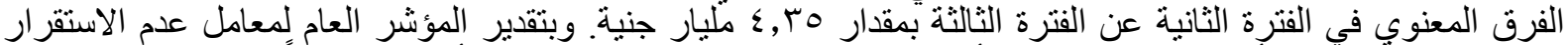

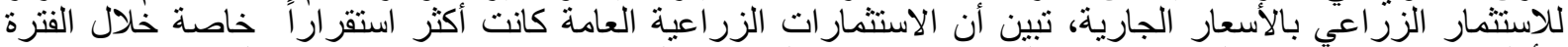

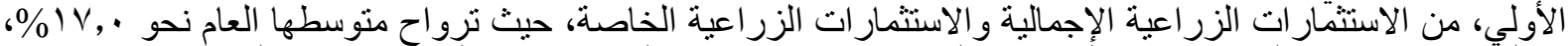

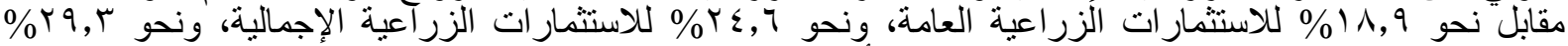

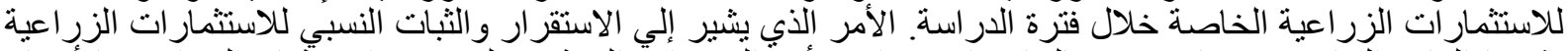

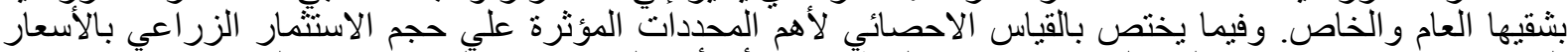

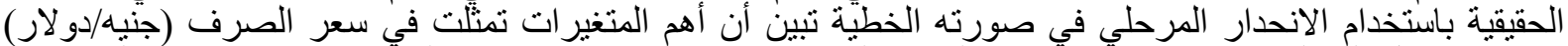

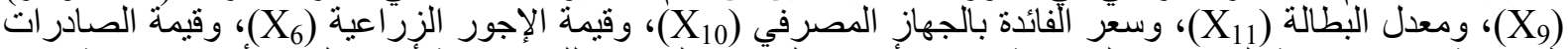

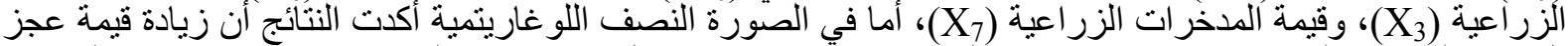

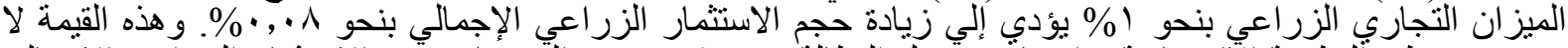

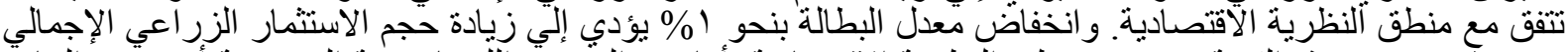

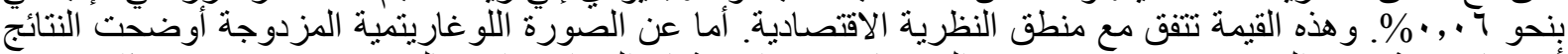

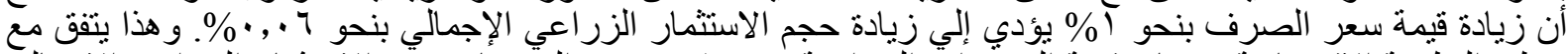

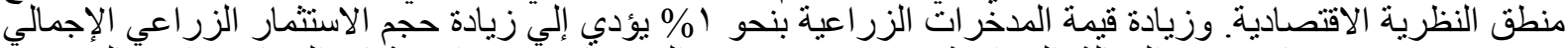

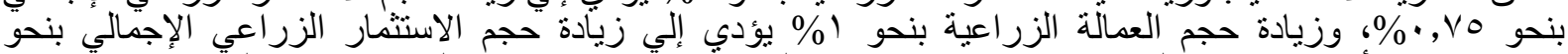

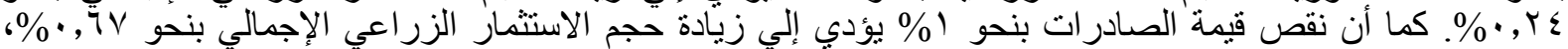

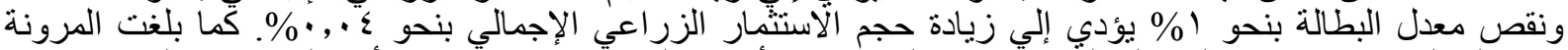

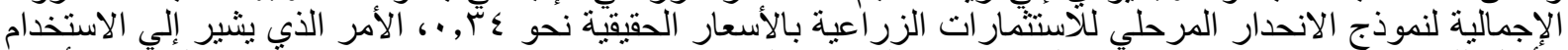

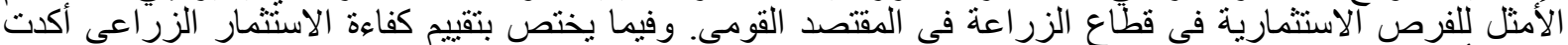

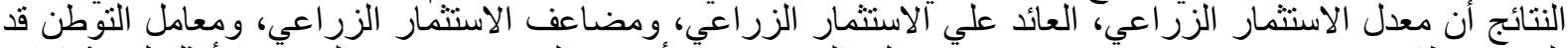

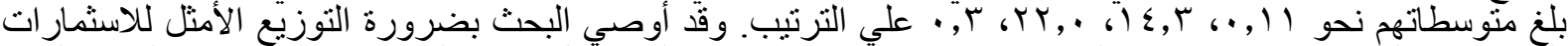

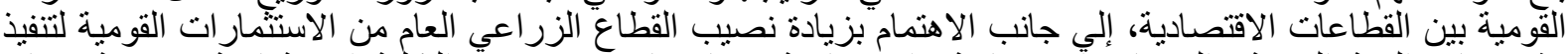

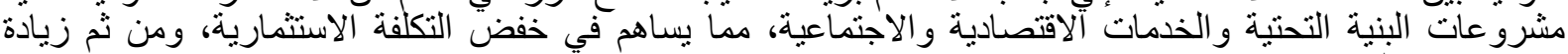
الاستثمار ات الزر اعية.

الكلمات الارشادية: كفاءة اقتصادية، محددات الاستثمار، كفاءة الاستثمار.

* Corresponding author: Tel.: +201060551139

E-mail address: wesam@aruedu.eg 


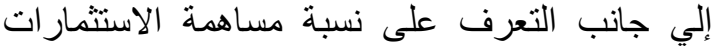
الزر اعية من إجمالي الاسنثمار ات القومية.

ץ_التحليل الاحصائي للعلاقات الانحدارية للاستثمار الزراعي بشقيه التعام والخاص علي ألئ أهم المحددات الاقتصادية المؤثره عليهم.

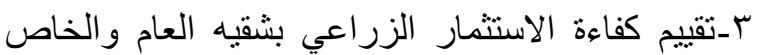

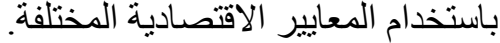

\section{أهمية البحث}

ترجع أهمية دراسة كفاءة الاستثمار الزراعي في

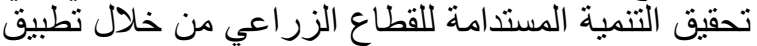

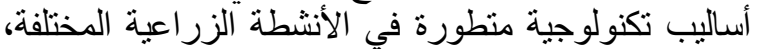

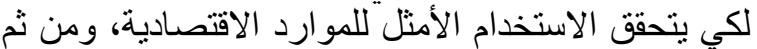

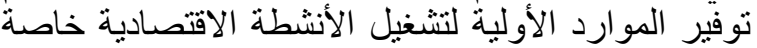

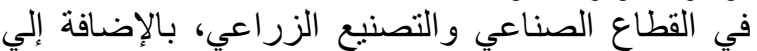

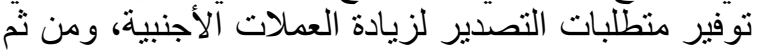

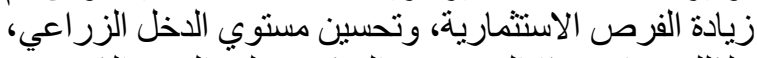

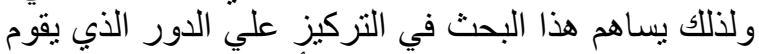

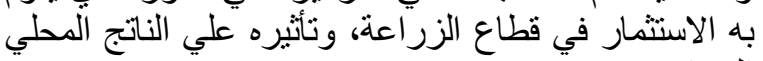

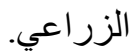

\section{الطريقة البحثية ومصادر جمع البيانات}

لتحقيق الأهداف سابقة الذكر، فقد اعتمد البحث علي

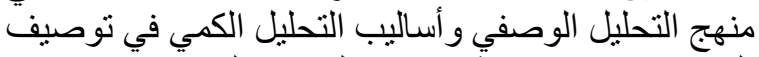

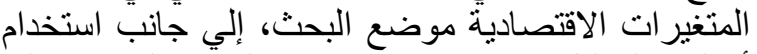

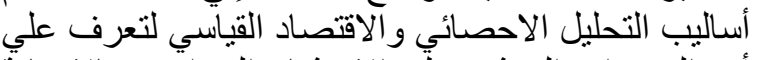

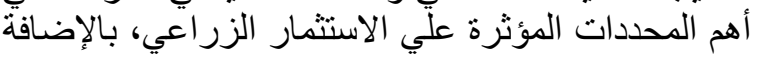

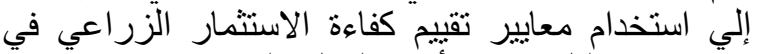

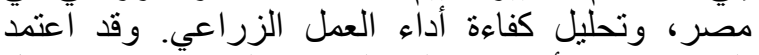

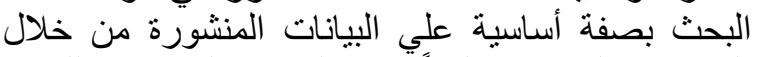

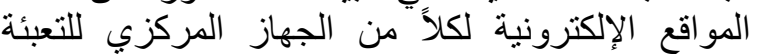

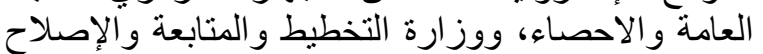

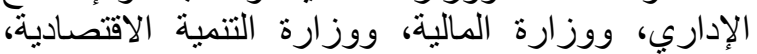

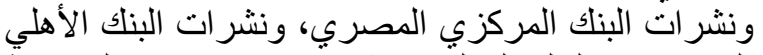

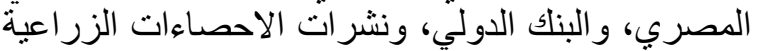

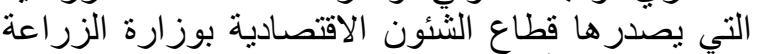

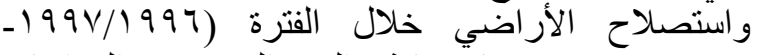

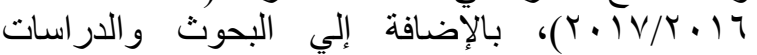
و الرسائل العلمية ذات الصات الإلة بموضوع الدر اسة.

\section{التثائج البحثبة}

الجاريع الراهن لقيمة الاستثمار الزراعي بالأسعار

تطور قيمة الاسثثمار الزراعي العام

باستعر اض الأرقام الواردة بجدول ال، يتبين أن قيمة الاستثمار الزراعي العام بالأسعار الجارية قد بلغت الإد حدها

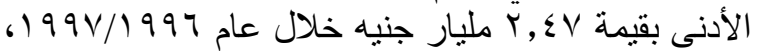

\section{المقدمة}

بعتبر القطاع الزراعي أحد الركائز الأساسية التي التي التئي

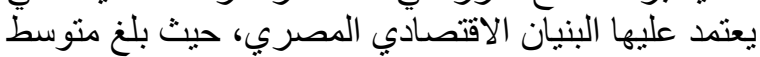

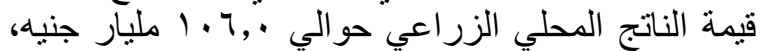

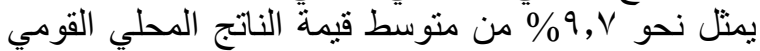

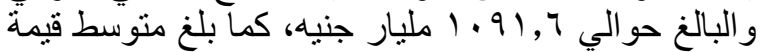

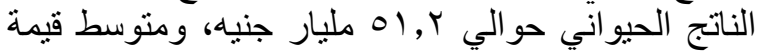

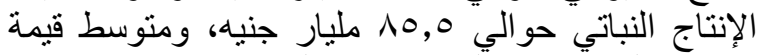

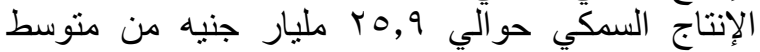

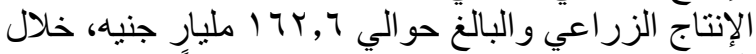

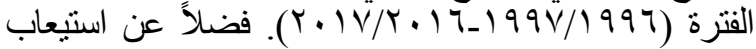

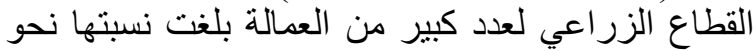

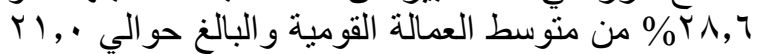

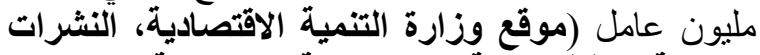

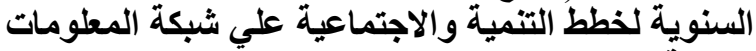

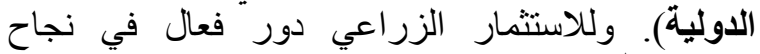

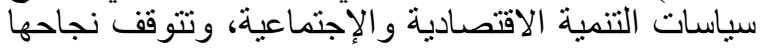

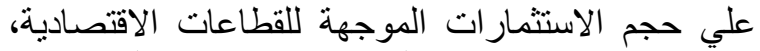

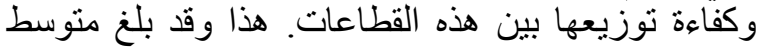

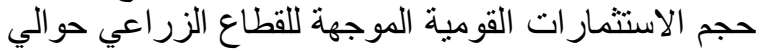

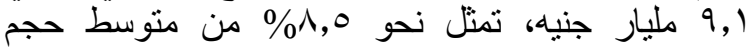

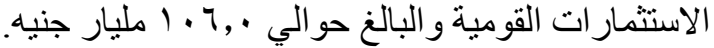

مشكلة البحث

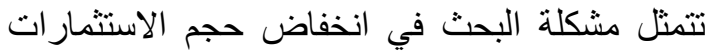

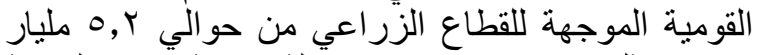

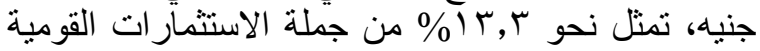

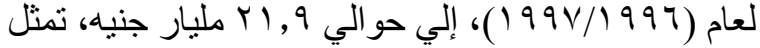

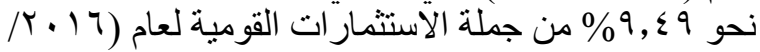
ع (Y P IV عليه انخفاض قدرة هذا القطاع علي زيادة معدلات الاكته الاكتفاء

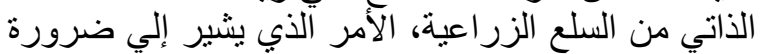

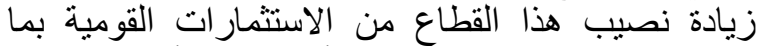

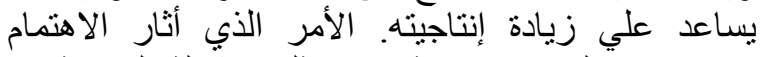

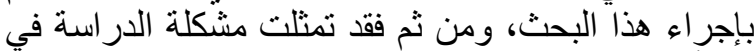

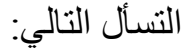

ا ـ ما هي المحددات و المتغير ات المؤثرة علي حجم الاستثمار

$$
\text { الزر آعي في مصر؟ المدوات }
$$

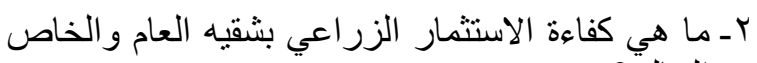

$$
\text { الحالي" ماني? }
$$

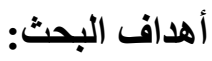

يهذف البحث بصفةً أساسية إلي التعرف علي كفاءة

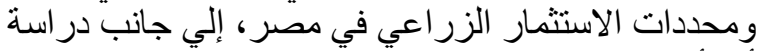

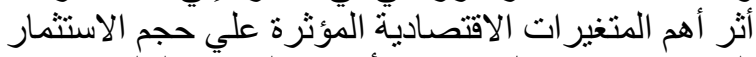
الزراعي، من خلال تحقيق الأهداف الفرعية التئ التالية:

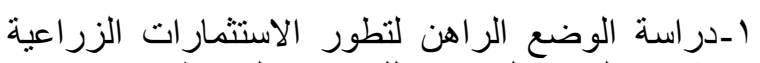

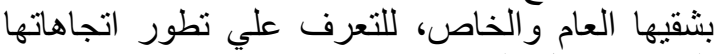

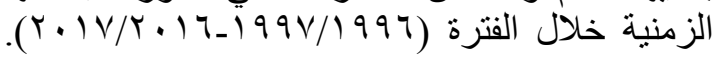


SINAI Journal of Applied Sciences (ISSN: 2314-6079), Vol. (8), Is. (1), Apr. 2019

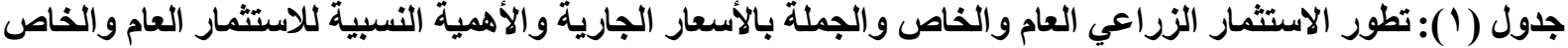

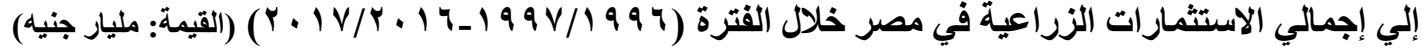

\begin{tabular}{|c|c|c|c|c|c|}
\hline \multirow[t]{2}{*}{ إجمالي الاستثمارات الزراعية } & \multicolumn{2}{|c|}{ الاستثمار الزراعي الخاص } & \multicolumn{2}{|c|}{ الاستثمار الزراعي العام } & \multirow[t]{2}{*}{ السنة } \\
\hline & $(\%)$ & جاري & $(\%)$ & جاري & \\
\hline 0,19 & OY, हl & $T, V Y$ & $\varepsilon \vee, 09$ & $T, \Sigma V$ & $1998 / 1999$ \\
\hline$\wedge, 17$ & $\varepsilon 7,79$ & $r, \wedge)$ & Or,M & $\varepsilon, r_{0}$ & $199 \wedge / 199 \vee$ \\
\hline$\Lambda, \leqslant Y$ & Aג, & $\varepsilon, O r$ & $\sum \neg, \mu r$ & r,q. & $1999 / 1991$ \\
\hline$\Lambda, 1 \mu$ & $7 ., 0 Y$ & $\varepsilon, 9 T$ & rq, ¿А & $r, Y)$ & $r \ldots / 19 q 9$ \\
\hline$\Lambda, r$. & $T \leqslant, V T$ & $0, r_{1}$ & ro, r & $r, \wedge 9$ & $r \ldots 1 / r_{\ldots} .$. \\
\hline 9,09 & 71,0 r & 0,9 . & 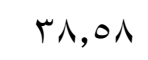 & $r, v$. & $r \ldots r / r \ldots 1$ \\
\hline ఛ, ¿. & $\varepsilon 9,79$ & $r, 11$ & $0 ., T_{1}$ & $r, Y r$ & $r \ldots r / r \ldots r$ \\
\hline$v, v r$ & 00,71 & $\varepsilon$ & $\leq \varepsilon, \varepsilon 1$ & $r, r q$ & م. الفترة الأولي \\
\hline $1 \wedge, \wedge$ & - & Y & - & $1 \wedge, \wedge$ & معامل التشتت النسبي \\
\hline$V, 07$ & or,q1 & $\varepsilon, \ldots$ & $\varepsilon \vee, \cdot q$ & $r, 07$ & $r \ldots \varepsilon / r \ldots r$ \\
\hline$\checkmark, \varepsilon r$ & $O V, Y \wedge$ & $\varepsilon, r_{O}$ & $\varepsilon Y, V Y$ & r,IV & $r \ldots \Delta / r_{\ldots} \ldots \varepsilon$ \\
\hline$\Lambda, \cdot \varepsilon$ & $70,1 \mathrm{~V}$ & $0, r \varepsilon$ & $r \varepsilon, \wedge r$ & $r, \wedge$. & $r \ldots r / r \ldots o$ \\
\hline$\vee, \vee q$ & $\neg \wedge, \wedge 1$ & 0, ( & 4,19 & $r, \varepsilon r$ & $r \ldots v / r \ldots r$ \\
\hline$\Lambda, \cdot \vee$ & $7 \varepsilon, 71$ & $0, Y r$ & ro,r & r, ^० & $r \ldots \wedge / r \ldots v$ \\
\hline $7, \wedge 7$ & $7 \cdot, \cdot 7$ & $\varepsilon, 1 Y$ & rq, q & $Y, V \varepsilon$ & $r \ldots q / r \ldots \wedge$ \\
\hline $7, \vee \leqslant$ & $O V, \sum Y$ & $\uparrow, \wedge \vee$ & $\varepsilon r, v r$ & $r, \wedge \wedge$ & $r \cdot 1 \cdot / r \ldots q$ \\
\hline$V, 7 r$ & $9 \cdot 9$. & $\varepsilon, \vee$. & rq, ir & r,qr & م. الفترة الثانية \\
\hline$v, \cdot$ & - & $1 \leqslant, 1$ & - & Ir, r & معامل التشتت النسبي \\
\hline$\checkmark, \wedge r$ & Or,Ir & $r, 07$ & $\varepsilon \wedge, \cdot r$ & $r, r \wedge$ & $r+11 / r+1$. \\
\hline $0, r V$ & $0 ., Y \Lambda$ & $r, V \cdot$ & $\varepsilon q, V Y$ & Y,TV & $r+\mid r / r+11$ \\
\hline$\Lambda, r \Lambda$ & $T \leqslant, \wedge$. & $0, \varepsilon r$ & $r_{0, Y}$. & $r, 90$ & $r+1 r / r+1 r$ \\
\hline חז, & $T \leq, r T$ & $V, 乏 \wedge$ & $r_{0,7 \Lambda}$ & $\varepsilon, 10$ & $r+1 \leq / r+1 r$ \\
\hline$|r, \Sigma|$ & 71,10 & $\Lambda, r$. & ґ, ^০ & $0, Y_{1}$ & $r+10 / r+1 \leq$ \\
\hline $17, Y \wedge$ & $79, \cdot \varepsilon$ & $11, r \varepsilon$ & $r \cdot, 97$ & $0, \cdot \varepsilon$ & $r+17 / r+10$ \\
\hline Y),人т & $V \vee, 90$ & $1 v, \cdot \varepsilon$ & $r, .0$ & $\varepsilon, \wedge r$ & $r+1 V / r+14$ \\
\hline $11,9 \vee$ & $\checkmark r, \wedge !$ & $v, 90$ & $r v, r_{l}$ & $\varepsilon, \cdot r$ & م.الفترة الثالثة \\
\hline$r \varepsilon, q$ & - & $\varepsilon \cdot$, & - & $r v, \cdot$ & معامل التشتت النسبي \\
\hline $9, .9$ & $\bullet q, \vee \vee$ & ט & $\varepsilon \cdot, r \varepsilon$ & $r, \varepsilon \varepsilon$ & المتوسط العام \\
\hline
\end{tabular}




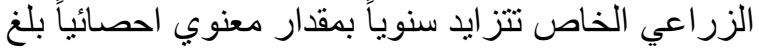

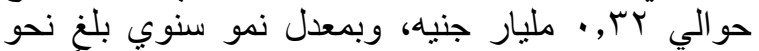
\%, Y

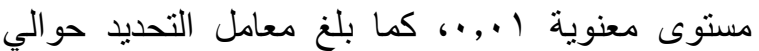

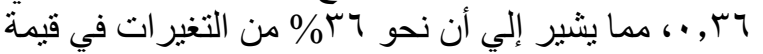

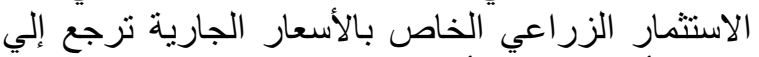

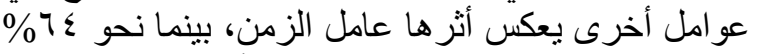

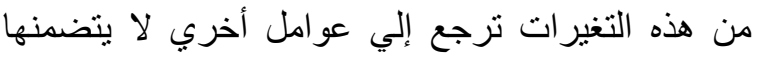

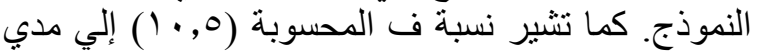

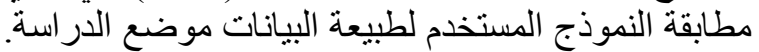

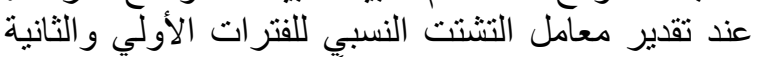
و الثالثة تبين أن نسبته كبيرة جداً بين الثناث فترات التهات، حيث

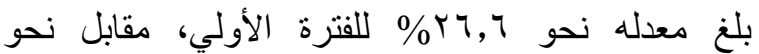

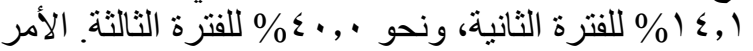

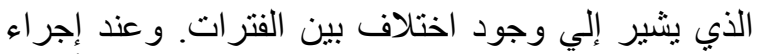

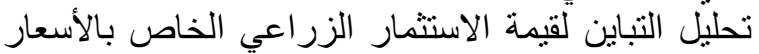

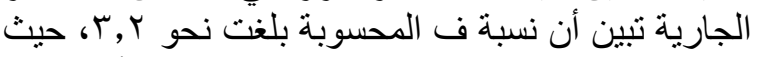

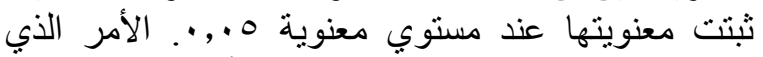

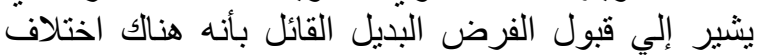

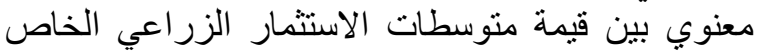

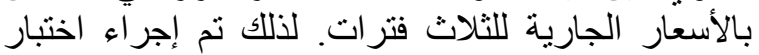

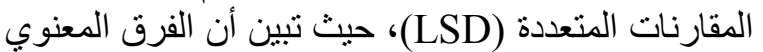

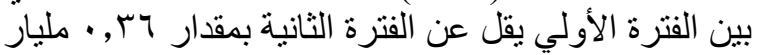
جنية وكذللك بقل الفترة الأولي بمقدار معنوي عن الفترة الفترة

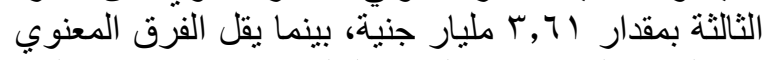

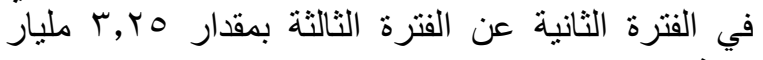

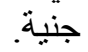

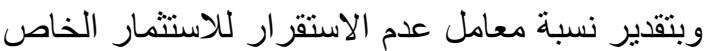

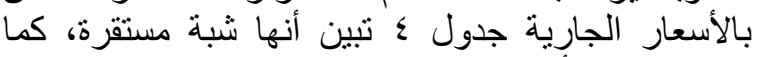

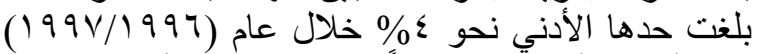

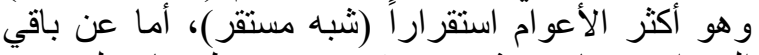

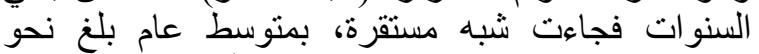

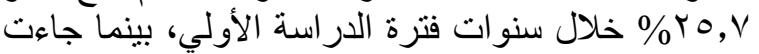

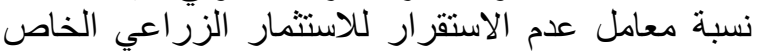

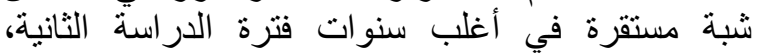

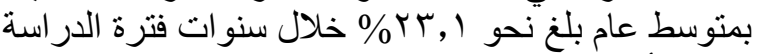

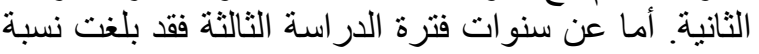

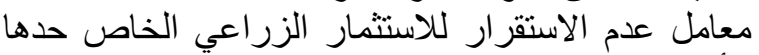

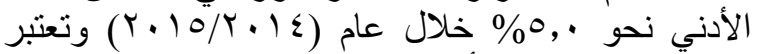

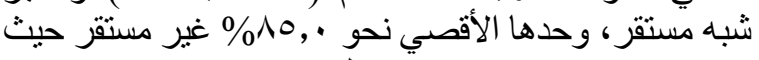

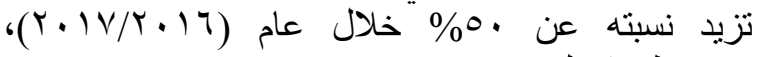

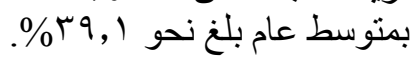

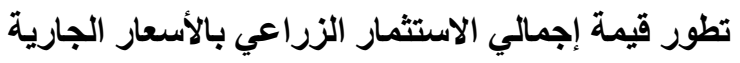

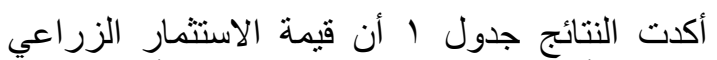

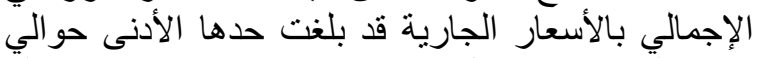

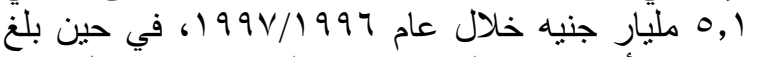

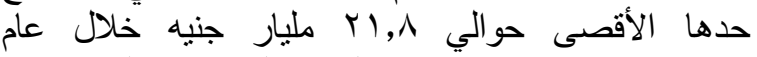

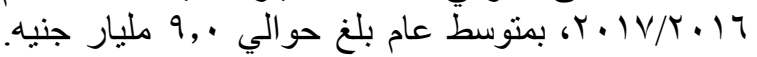

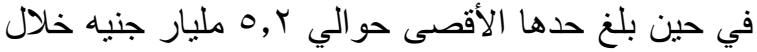

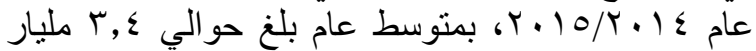

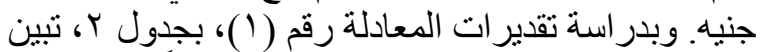

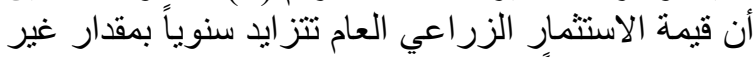

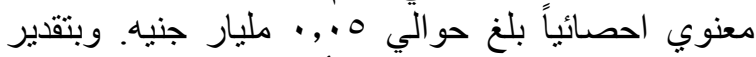

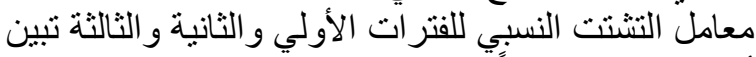
أن نسبته كبيرة جداً بين الفترتين الثانية و الثنالثة، ومتباينة

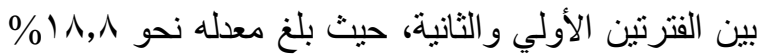

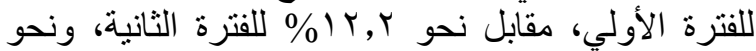

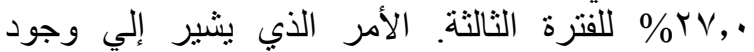
اختلاف بين الفترات. و وعليه فقد تم إجراء تحليل التئل التباين لقيمة الاستثمار الزراعي العام بالأسعار (ANOVA)

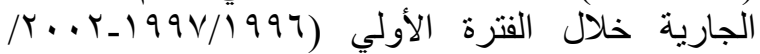

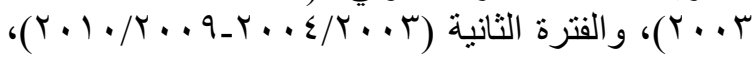

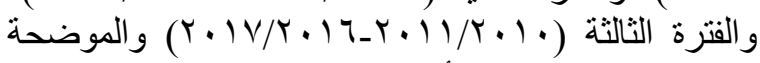

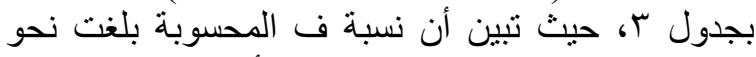

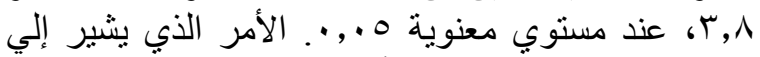

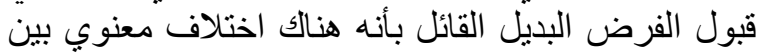

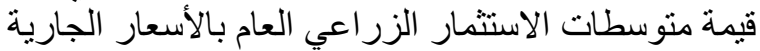

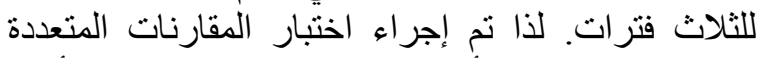

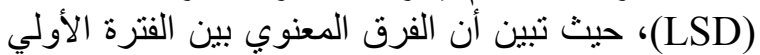

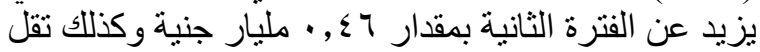
الفترة الأولي بمقدار معنوي عن الفنارة الفترة الثالثة بمقدار سا,، مليار جنية، بينما بقل الفرق الفئل المعنوي في الفترة الثانية عن الفترة الثالثة بمقدار 9 . , الئيار جنية.

وعند تقدير نسبة معامل عدم الاستقرار للاستثمار

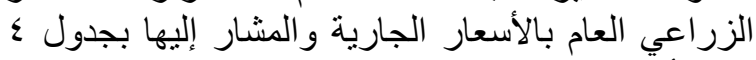

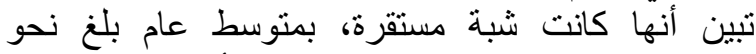

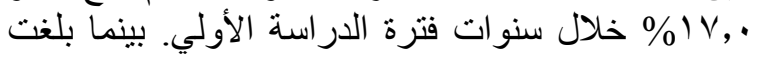

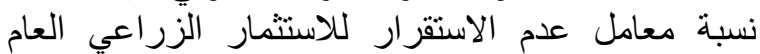

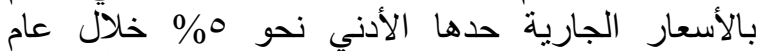

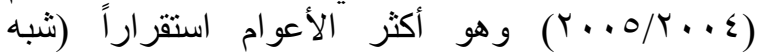

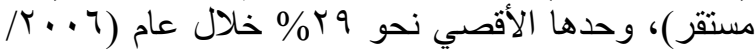

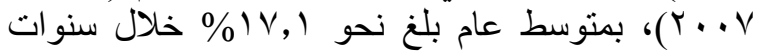

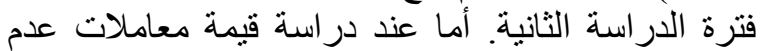

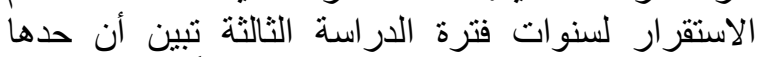

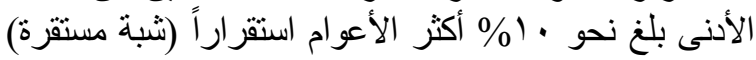

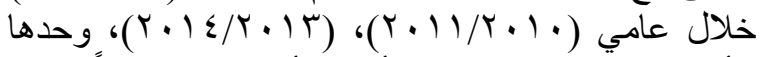

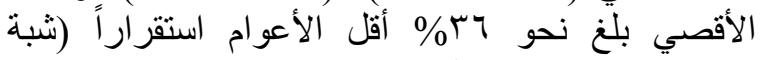

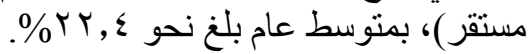

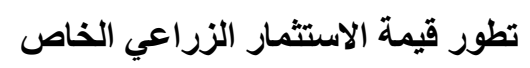

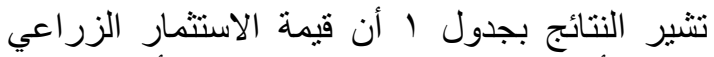

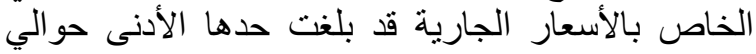

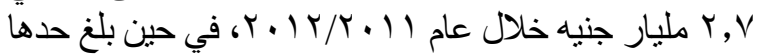

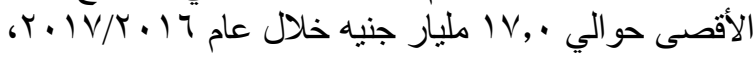

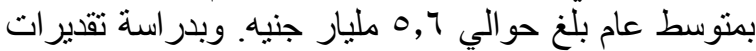

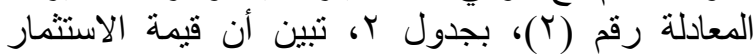




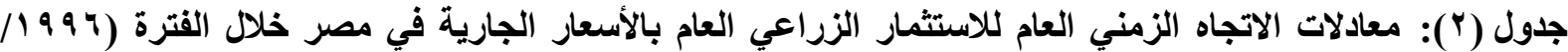

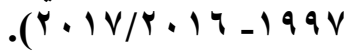

\begin{tabular}{|c|c|c|c|c|c|}
\hline المحسوبة & $\overline{\mathbf{R}^{2}}$ & $\begin{array}{c}\text { النمو السنوي } \\
\text { (\%) }\end{array}$ & معادلة الاتجاه الزمني العام م & المتغيرات الاقتصادية & المعادلة \\
\hline 3.17 & 0.14 & 1.5 & $\begin{aligned} & \mathbf{Y}_{\mathbf{i}}= 2.88+0.05 \mathbf{T}_{\mathbf{i}} \\
&(7.99)^{* *}(1.78)\end{aligned}$ & $\begin{array}{c}\text { General Agricultural } \\
\text { Investment (LE billion) }\end{array}$ & 1 \\
\hline $10.56^{* *}$ & 0.36 & 5.7 & $\mathbf{Y}_{\mathbf{i}}=\underset{(1.76) \quad}{2.51+0.32 \mathbf{T}_{\mathbf{i}}}$ & $\begin{array}{l}\text { Private agricultural investment } \\
\text { (LE billion) }\end{array}$ & 2 \\
\hline $9.79^{* *}$ & 0.34 & 4.1 & $\begin{array}{r}\mathbf{Y}_{\mathbf{i}}=5.03+0.37 \mathbf{T}_{\mathbf{i}} \\
(3.42)^{* *}(3.13)^{* *}\end{array}$ & $\begin{array}{c}\text { Total Agricultural Investment } \\
\text { (LE billion) }\end{array}$ & 3 \\
\hline
\end{tabular}

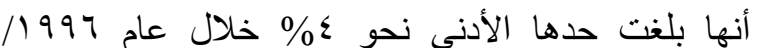

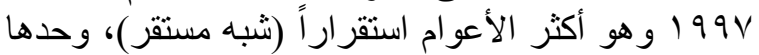

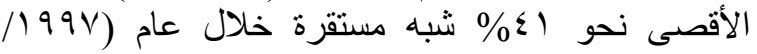

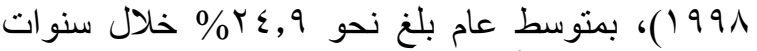

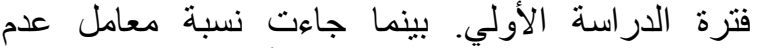

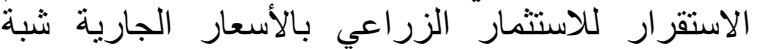

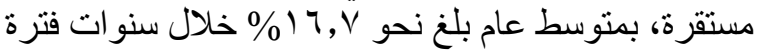

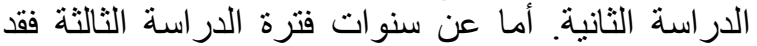
بلغت نسبة معامل عدم الاستقرار للاستثمار الزئة الزراعي

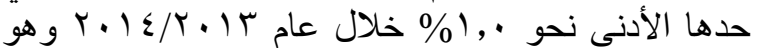

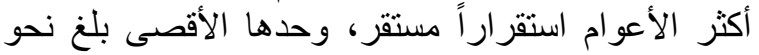

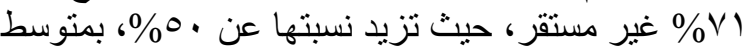

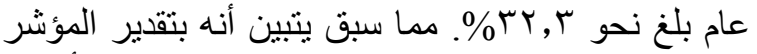

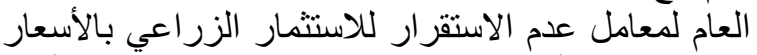
الجارية، تبين أن الاستثمار التئمار الزراعية العامة العامة كانت أكثر

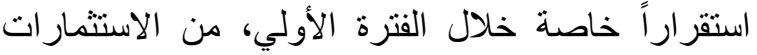

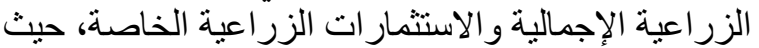

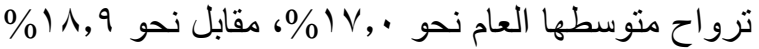

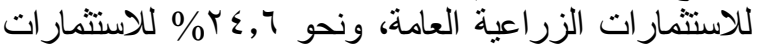

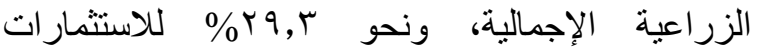

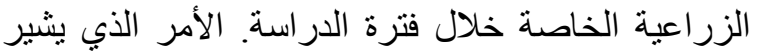
إلي الاستقرار و الثبات النسبي للاستثمارات الزية الزية الزية

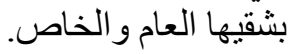

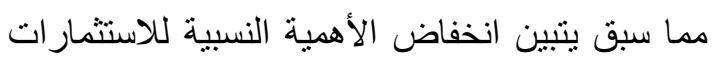

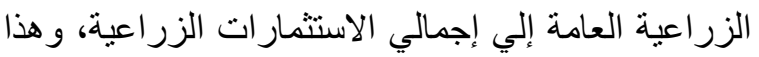

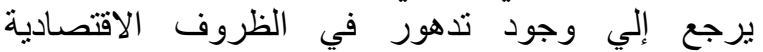

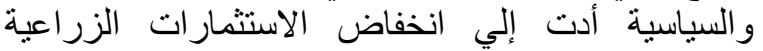

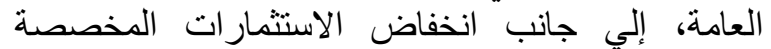
للقطاع الزراعي العام بالنسبة إلي القطاع الزراعي

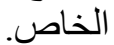

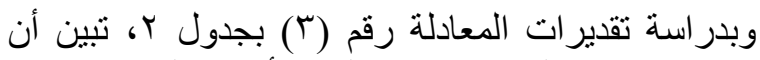

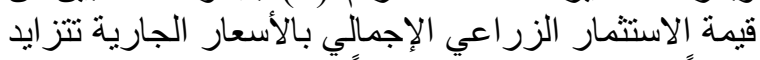

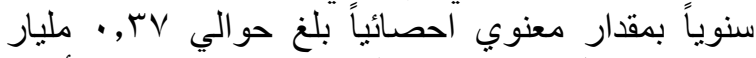

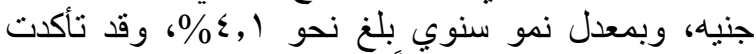

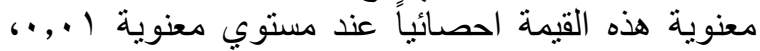

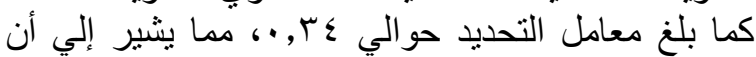

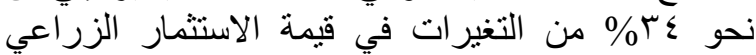

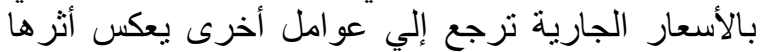

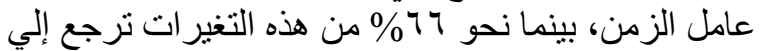

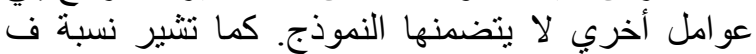

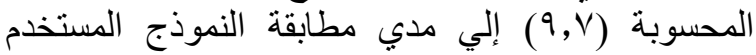

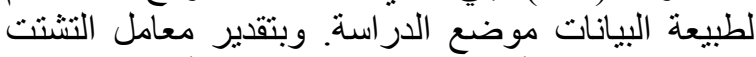

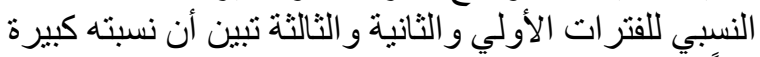

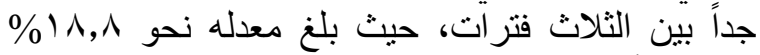

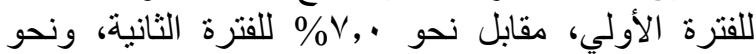

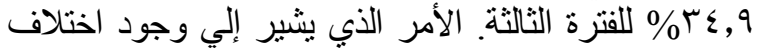
بين الفترات. وعند إجراء تحلئ الاعليل التباين لقيمة الاستثمار

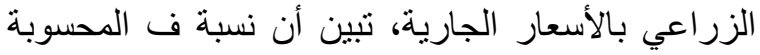

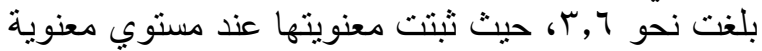

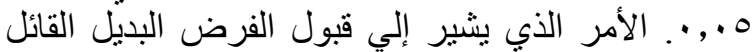

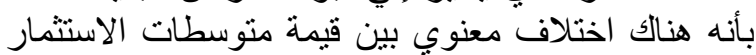

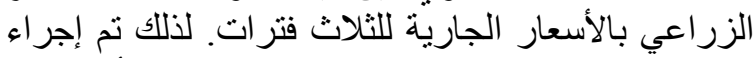

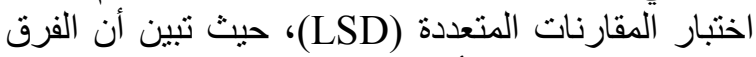

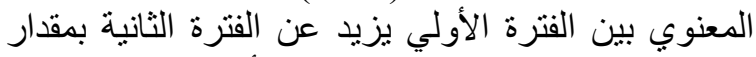

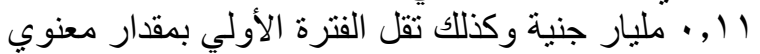

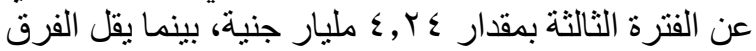
المعنوي في الفترة الثانية عن الفترة الثالثة بمقدار هـرــ

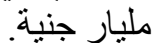

وبدراسة نسبة معامل عدم الاستقرار للاستتمار الزراعي بالأسعار الجارية والمشار إليها بجدول ع تبين 
جدول (r): نتائج تحليل التباين (ANOVA) واختبار المقارنات المتعددة (LSD) للاستثمار الزراعي العام والخاص

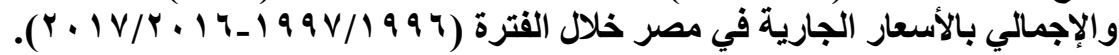

\begin{tabular}{|c|c|c|c|c|}
\hline \multicolumn{3}{|c|}{ اختبار المقارنات المتعددة (LSD) } & \multirow[t]{2}{*}{ اختبار التباين (F) } & \multirow[t]{2}{*}{ البيان } \\
\hline الثانية & الأولي & الفترة & & \\
\hline $0.46^{*}$ & $\begin{array}{l}0.63 \\
1.09^{*}\end{array}$ & الأأولية & $3.89^{*}$ & الاستثمار الزراعي العام (المليار جنيه) \\
\hline $0.24^{*}$ & $\begin{array}{l}3.61^{*} \\
3.37^{*}\end{array}$ & الأاولية & $3.26^{*}$ & الاستثمار الزراعي الخاص (المليار جنيه) \\
\hline $0.23^{*}$ & $\begin{array}{l}4.24^{*} \\
4.47^{*}\end{array}$ & الأأولية & $3.69^{*}$ & إجمالي الاستثمارات الزراعية (المليار جنيه) \\
\hline
\end{tabular}

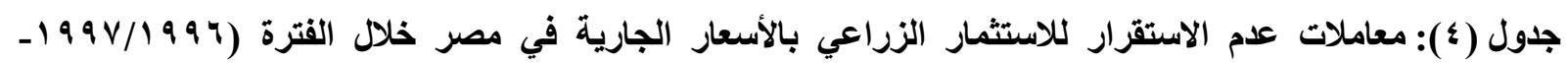
( r. IV/r + 17

\begin{tabular}{|c|c|c|c|}
\hline \multicolumn{3}{|c|}{ معاملات عدم الاستقزار لاستثمار الزراعي الجاري (\%) } & \multirow[t]{2}{*}{ 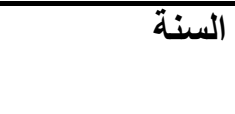 } \\
\hline جملة & 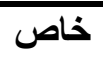 & عام & \\
\hline$\varepsilon$ & $\varepsilon$ & 17 & $199 \mathrm{~V} / 1999$ \\
\hline «1 & r) & $\leq 7$ & $199 \wedge / 199 \vee$ \\
\hline rv & r. & rq & $1999 / 1991$ \\
\hline ro & r. & $\varepsilon$ & $r \ldots / 199 q$ \\
\hline 19 & rq & $\wedge$ & $r \ldots 1 / r \ldots$ \\
\hline rt & 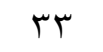 & 17 & $r \ldots r / r \ldots 1$ \\
\hline 17 & r & • & $r \ldots r / r \ldots r$ \\
\hline$r \leq, q$ & $r \bullet, r$ & IV,. & م. الفترة الأولي \\
\hline 0 & r) & 9 & $r \ldots \varepsilon / r \ldots r$ \\
\hline 11 & r) & ○ & $r \ldots \Delta / r_{\ldots} \ldots \varepsilon$ \\
\hline$\wedge$ & $\wedge$ & iv & $r \ldots r / r \ldots \theta$ \\
\hline $1 \varepsilon$ & 11 & rq & $r \ldots V / r \ldots r$ \\
\hline 10 & 11 & 11 & $r \ldots \Lambda / r \ldots v$ \\
\hline r. & rᄉ & rt & $r \ldots q / r \ldots \Lambda$ \\
\hline$r \varepsilon$ & $\leqslant 0$ & $r$. & $r \cdot 1 \cdot / r \ldots q$ \\
\hline $17, V$ & $r r, l$ & $1 v, 1$ & م. الفترة الثانية \\
\hline ro & 01 & 1. & $r+11 / r \cdot 1$. \\
\hline 01 & 70 & YV & $r+1 r / r+11$ \\
\hline r & r & YI & $r+1 r / r+1 r$ \\
\hline 1 & 1. & 1. & $r+1 \leqslant / r+1 r$ \\
\hline 11 & 0 & צ r & $r+10 / r+1 \leq$ \\
\hline r & ז & $r$. & $r+17 / r+10$ \\
\hline v) & 10 & rT & $r+1 V / r+17$ \\
\hline$r r, r$ & $r q, 1$ & $r Y, \varepsilon$ & م. الفترة الثالثة \\
\hline$r \leq, 7$ & $r q, r$ & 11,9 & م. المتوسط العام \\
\hline
\end{tabular}




\section{الصورة النصف اللوغاريتمية}

تبين نتائج المعادلة رقم (0) بجدول ه ه أن الدالة المقدرة

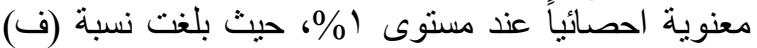

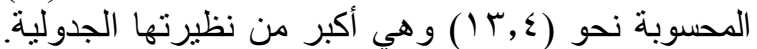

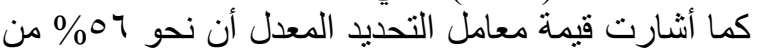

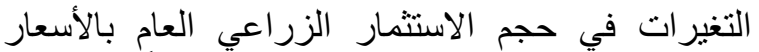

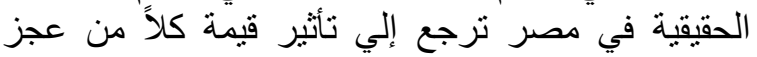

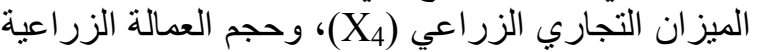

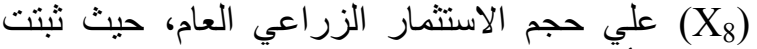

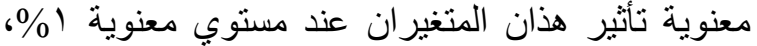

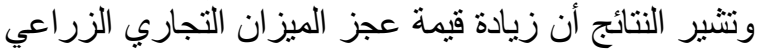

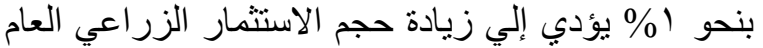

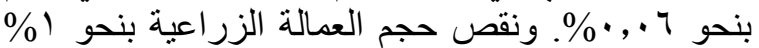

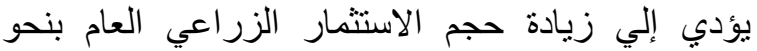

r ا , •\%. و هذا لا يتفق مع منطق النظرية الاقتصادية.

\section{الصورة اللوغاريتمية المزدوجة}

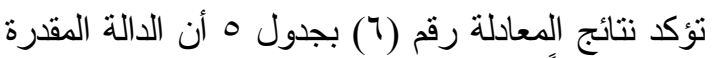

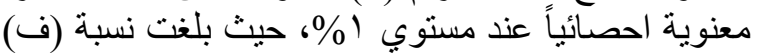

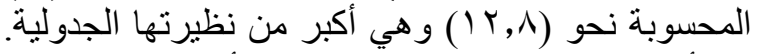

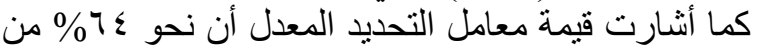
التغيرات في حجم الاستثمار الزراعي التئي العام بالأسعار

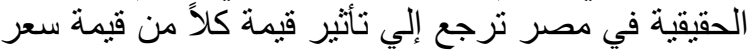
الصرف (جنيه/دولار) (X)(X)، وسعر الفيائدة علي الودائع

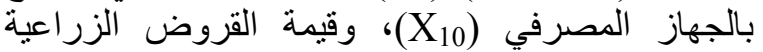

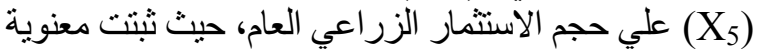

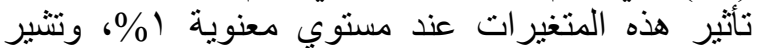

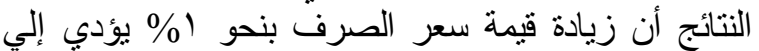

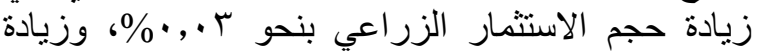

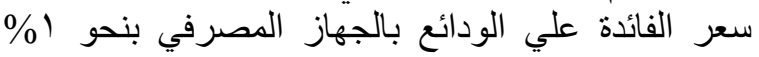

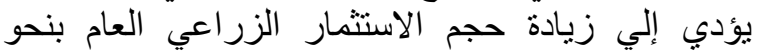

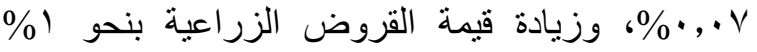

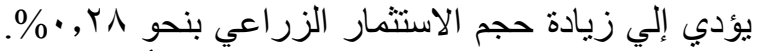

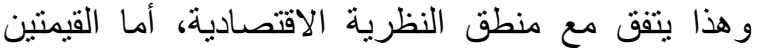

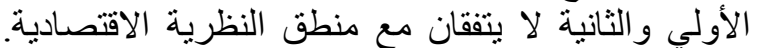

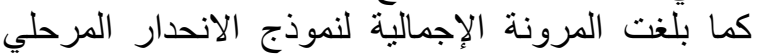

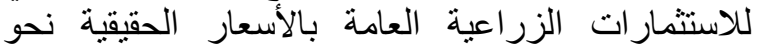

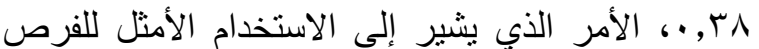

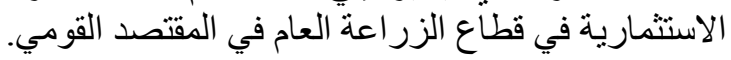

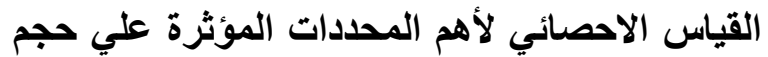 الاستثمار الزراعي الخاص الاصم الفمائ}

الصورة الخطية

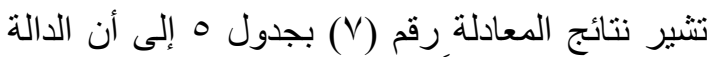

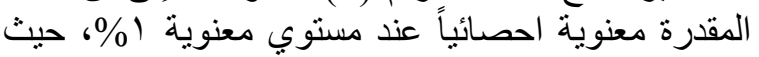

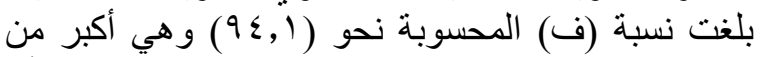
نظيرتها الجدولية. كما تتشير قيمة معامل التحديد المعدل أن الن الني

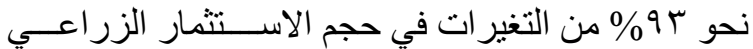

القياس الإحصائي لأهم المحددات المؤثرة علي حجم الاستثمار الزراعي في مصر الاحمر الاعد

القياس الاحصائي لأهم المحددات المؤثرة علئي الزمبر حجم الاستثمار الزراعي العام الام المائ

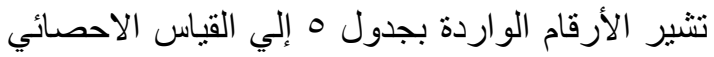

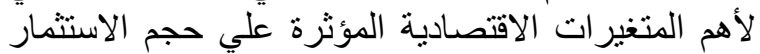

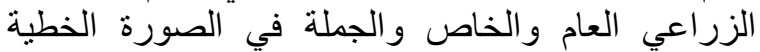

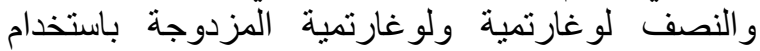

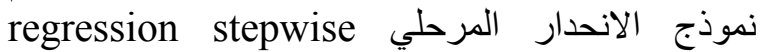

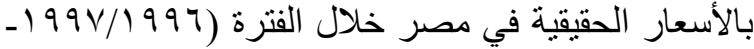

( $(T \cdot) V / T \cdot 1 T$

الصورة الخطية

أظهرت نتائج المعادلة رقم (ع) بجدول ه أن الدوبة الدالة

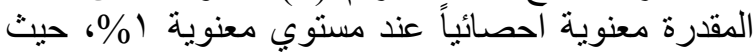

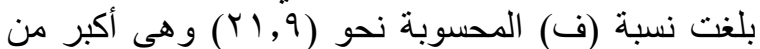

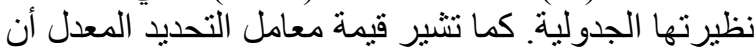

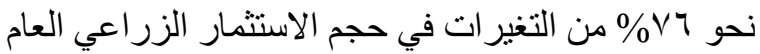

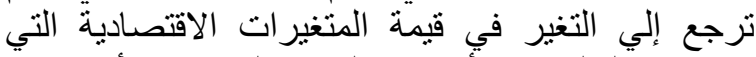

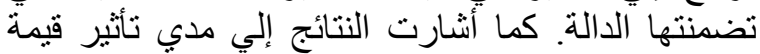

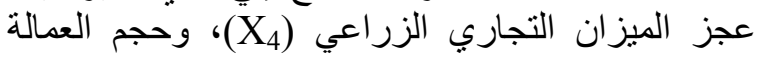

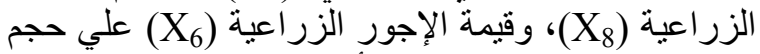

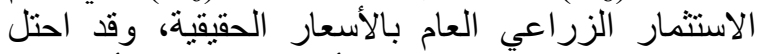

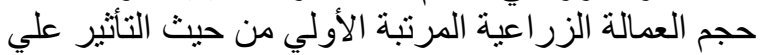

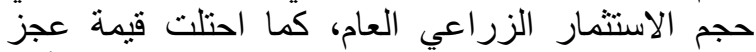

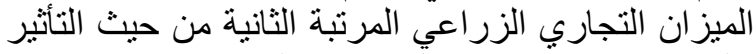

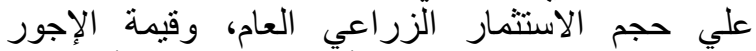

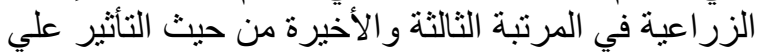

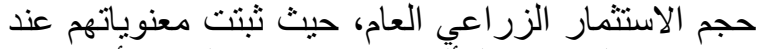

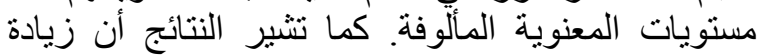

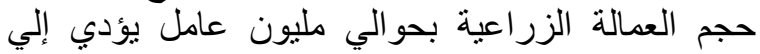

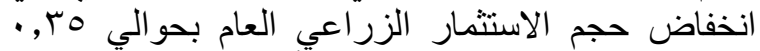
مليار جنيه، وقد يعزي ذلإضك إلي أن زيادي الزيام حجم العمالة

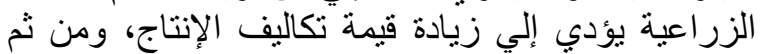

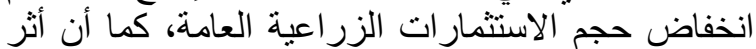

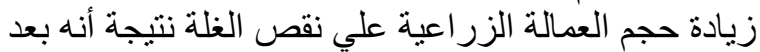

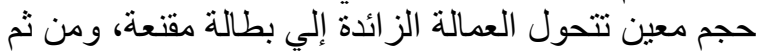

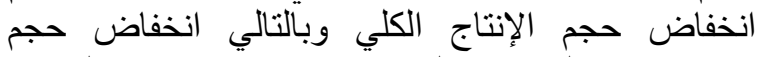

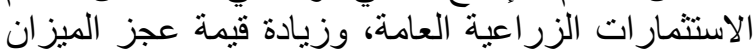

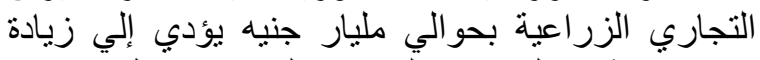

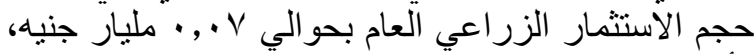

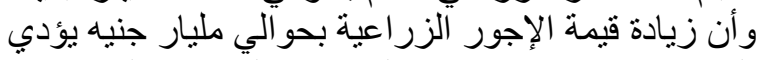

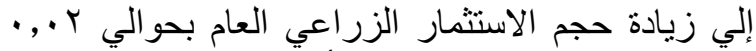
مليار جنيه. وتشير زيادة قيمة الأجور الزراعية الاعية إلي زيادة الإلية تكاليف الإنتاج، ومن ثم انخفاض الغلة الغالة الإنتاجية، نتيجة

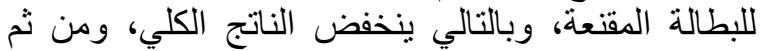
انخفاض الاستثمار ات الزّر اعية العامة، وهذا لا لا يتفق مع لع

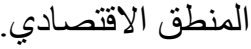


جدول (0): القياس الإحصائي لأهم المتغيرات الاقتصادية المؤثرة علي حجم الاستثمار الزراعي بالأسعار الحقيقية

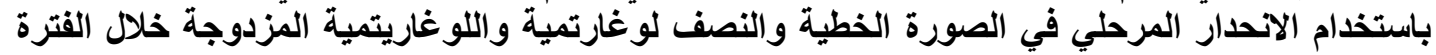
( $(r+1 V / r+17-199 V / 1997)$

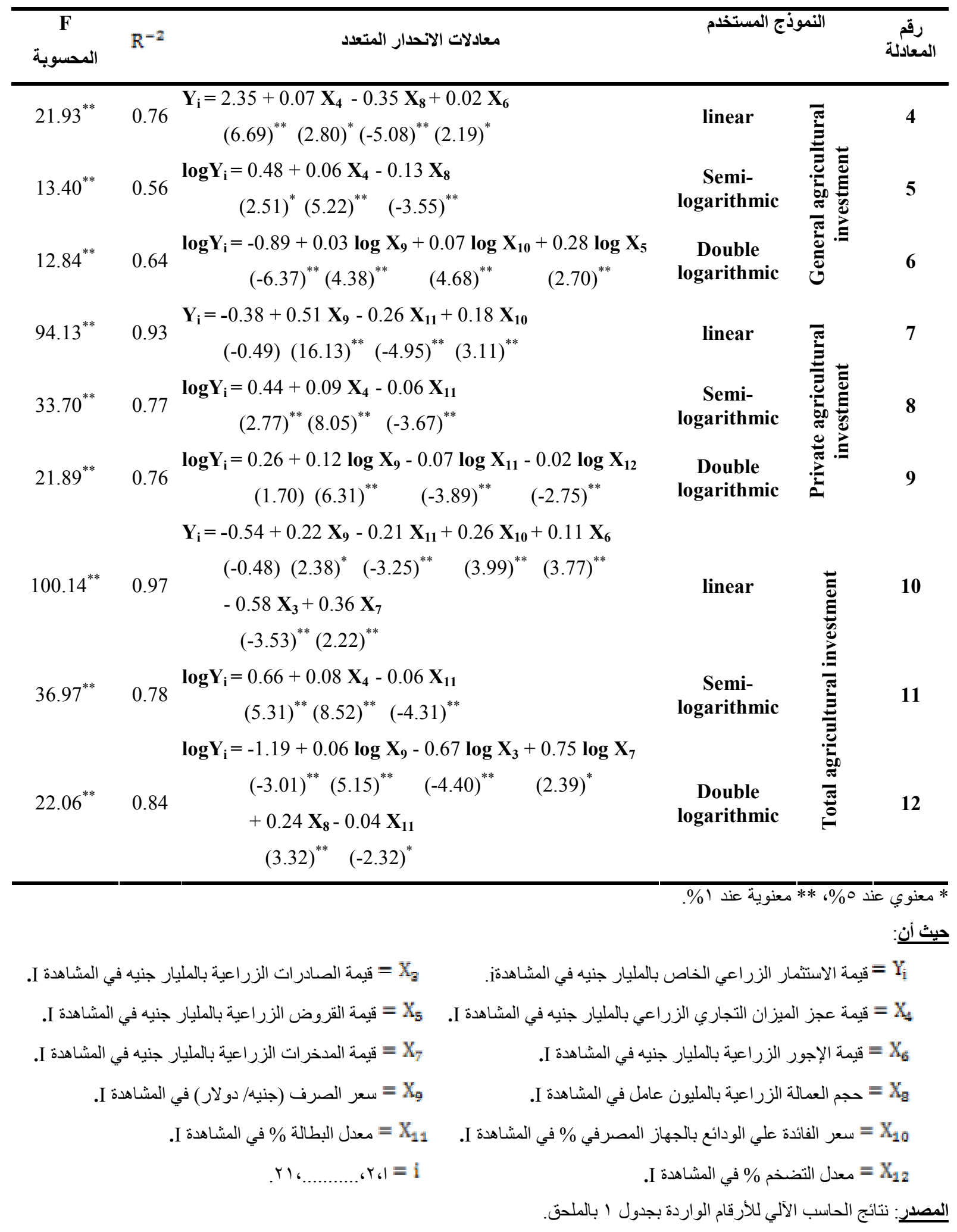




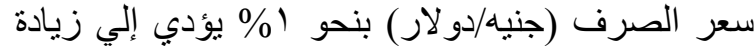

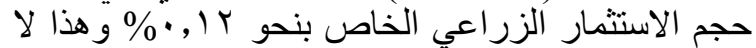

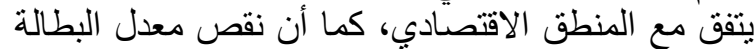

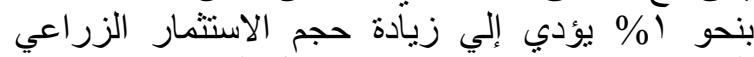

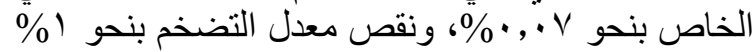

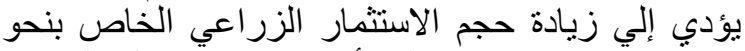

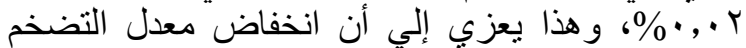

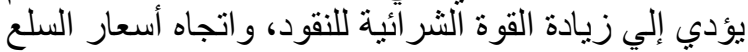

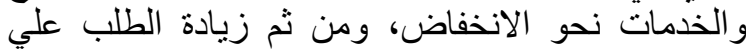

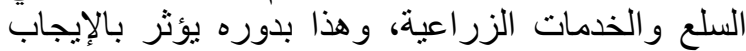

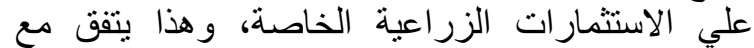

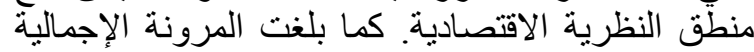

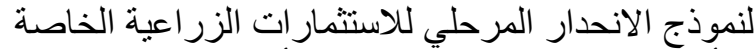

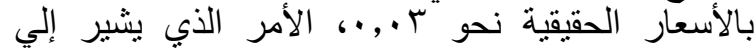

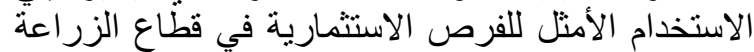
الخاص في المقتصد القومي.

\section{القياس الاحصائي لأهم المحددات المؤثرة علي حجم الأمي

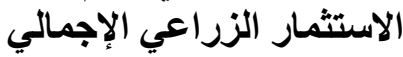

الصورة الخطية

تشير نتائج المعادلة رقم (• (1) بجدول ه إلى أن الدالة

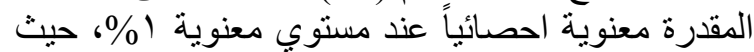

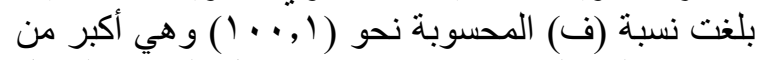

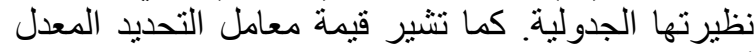

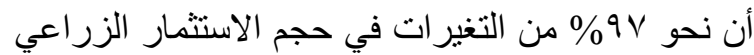

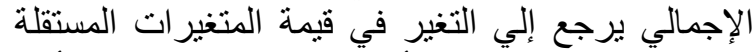
التي تضمنتها الدالة. كما أشنارت النتائج إلي مدي تأثير الثير

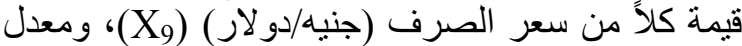

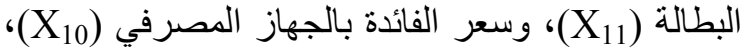

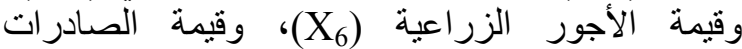

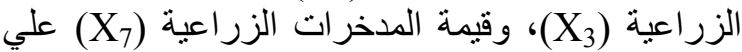

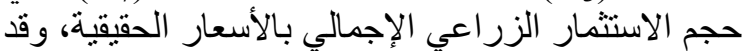

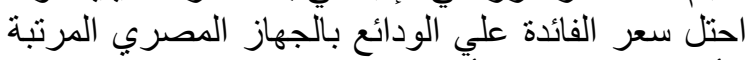

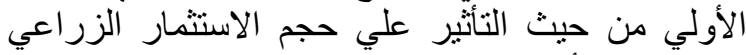

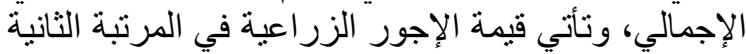

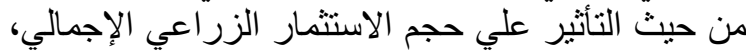

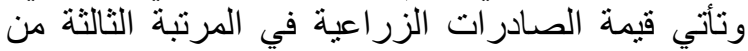

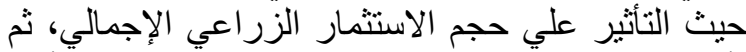
يأتي معدل البطالة في المرتبة الرابعة من حيث الزيث التأثير علي حجم الاستثمار الزراعي الإجمالية الرابعة ويني هيأتي سعر التير

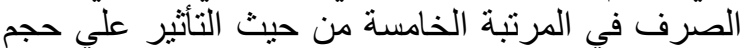
الاستتمار الزراعي الإجمالي، ثم تأتي قيمة التئية المدخرات

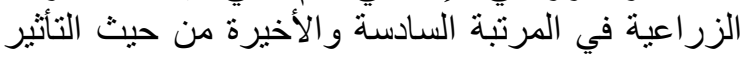

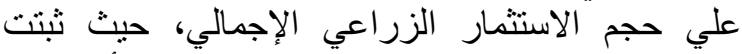

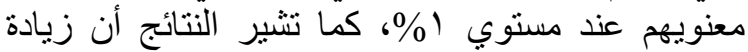

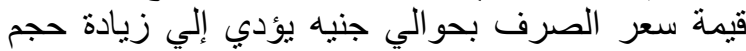

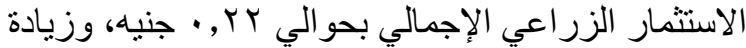

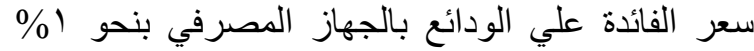
يؤدي إلى زيادة حجم الاستثمار الزراعي الإجمالي
الخاص ترجع إلي التغير في قيمة المتغير ات المستقلة التي

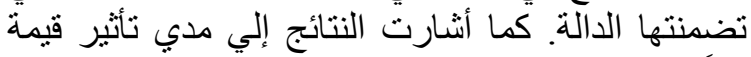

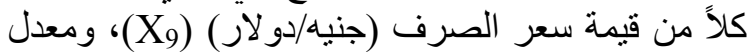

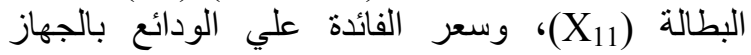
المصرفي (X)

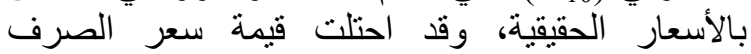

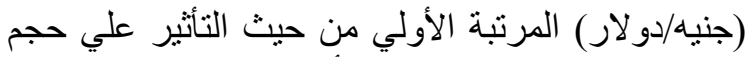

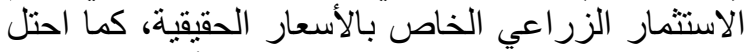

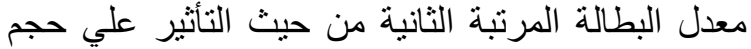

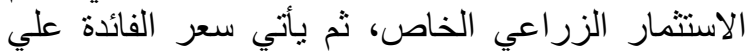

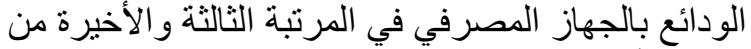

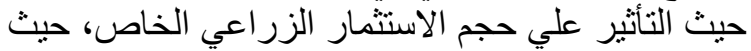

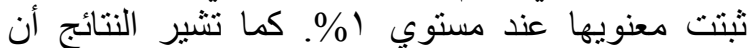

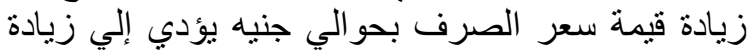

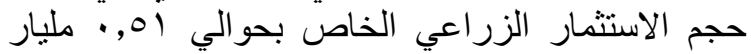

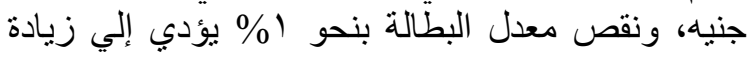

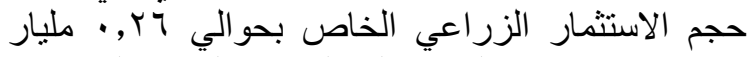

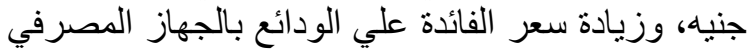

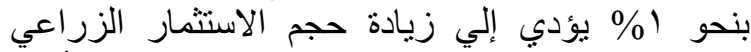

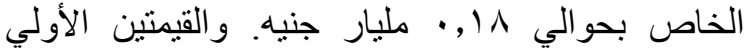
و الأخيرة لا تتفق مع المنطق الاقتصادي.

\section{الصورة النصف لوغاريتمية}

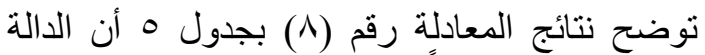

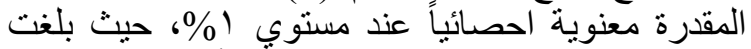

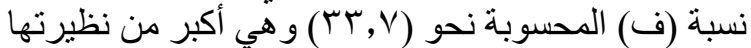

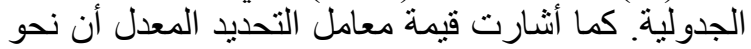

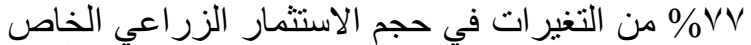

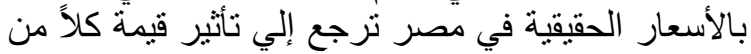

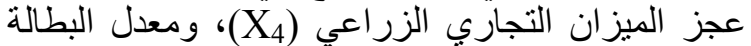

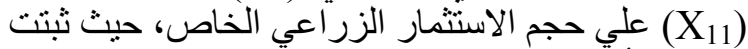

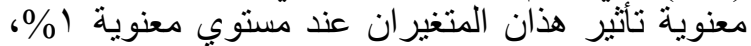

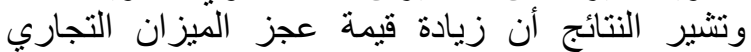

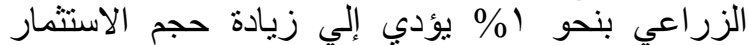

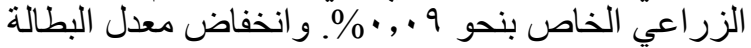
بنحو الزو بؤدي إلى زيادة حجم الاستثمار الزراعي

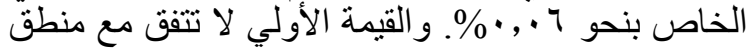
النظرية الاقتصادية.

\section{الصورة اللوغاريتمية المزدوجة}

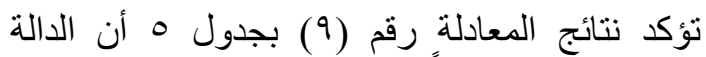

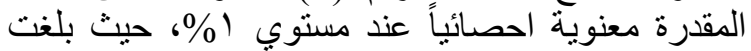

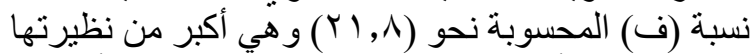

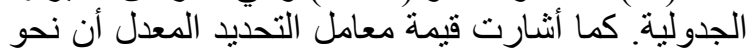

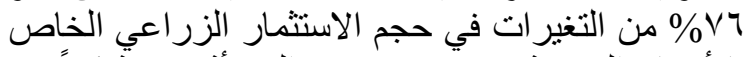

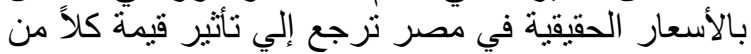

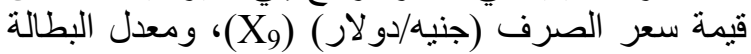
(X)

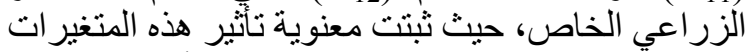
عند مستّى معنوية (\%)، وتثير النتائج أن زيادة قيمة النية 


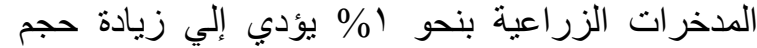

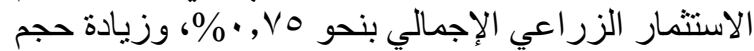

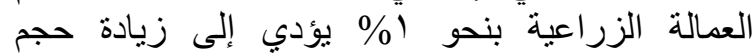

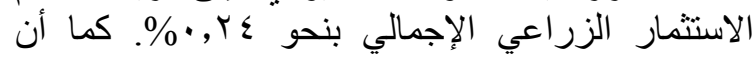

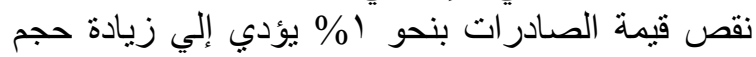

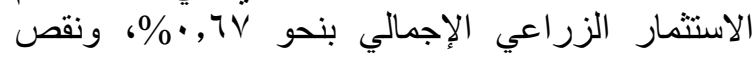

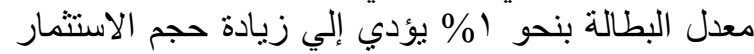

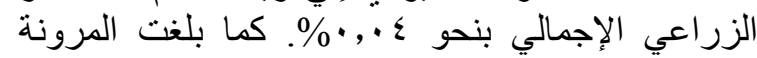

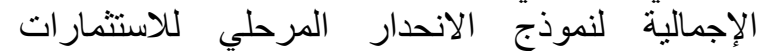

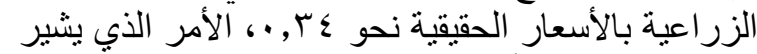

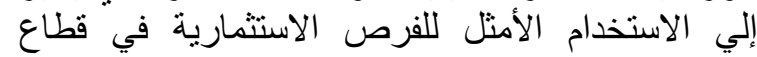
الزر اعة في المقتصد القومي.

\section{تقييم كفاءة الاستثثار الزراعي في مصر باستخدام} المعاييز الاقتصادية

تشير الأرقام الواردة بجدول 7 إلى معايير قياس كفاءة

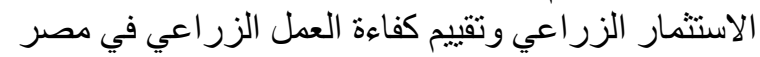

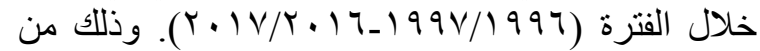

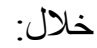

\section{معدل الاستثمار الزراعي}

توضح النتائج أن معدل الاسثنمار الزراعي الإجمالي

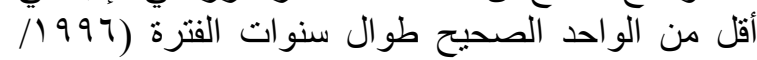

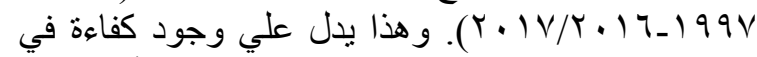

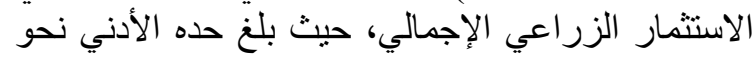

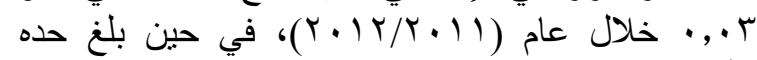

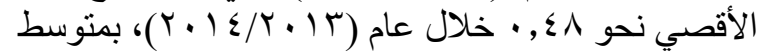

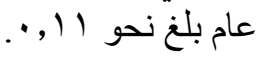

\section{العائد علي الاستثمار الزراعي (إنتاجية الاستثمار)}

تبين النتائج أن العائد علي الاستثمار الزراعي الإجمالي

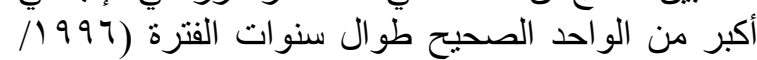

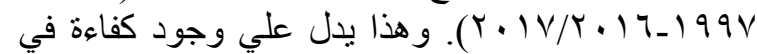

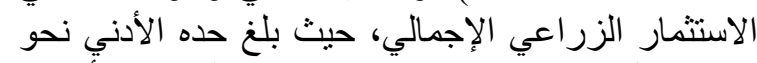

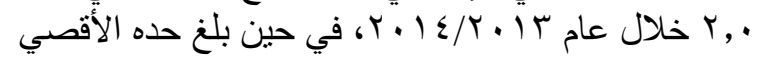

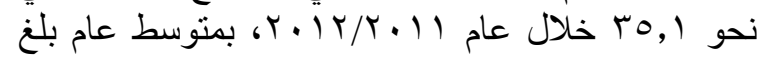

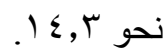

\section{مضاعف الاستثمار}

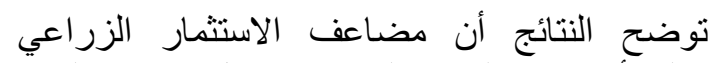

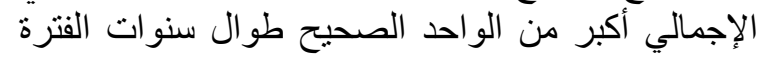

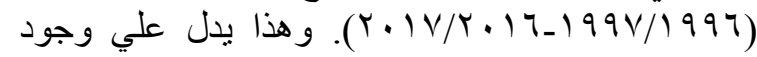

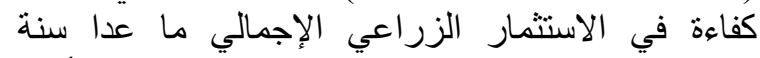

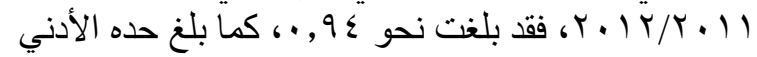

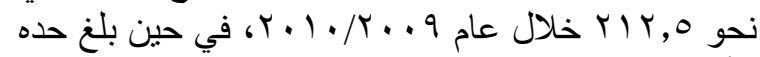

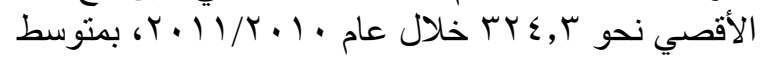

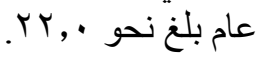

بحوالي جاب, •جنيه، وزيادة قيمة الأجور الزراعية بحوالي

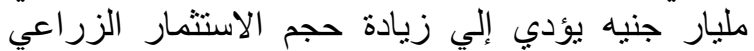

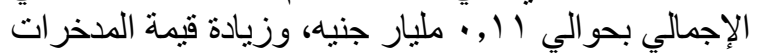

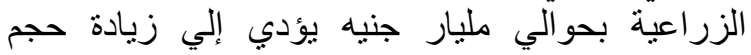

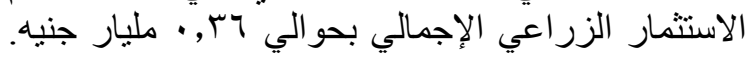

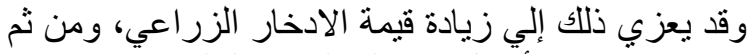

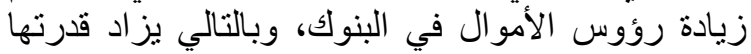

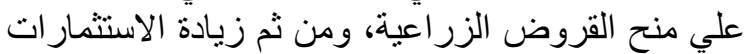

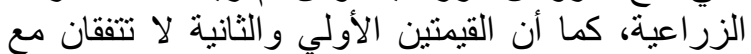

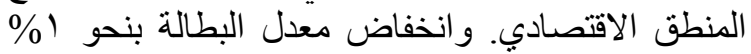

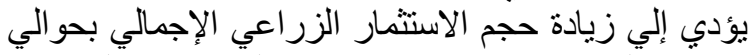

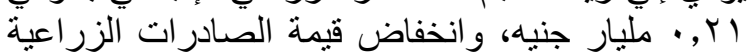

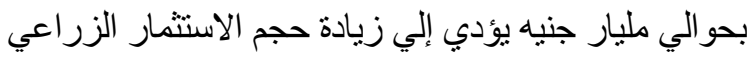

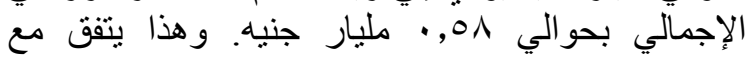
المنطق الاقتصادي.

\section{الصورة النصف لوغاريتمية}

تؤكد نتائج المعادلة رقم (1 (1) بجدول ه أن الدالة المقدرة

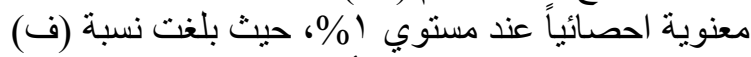

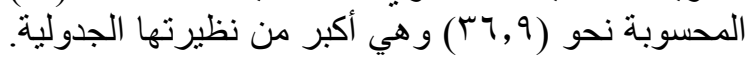

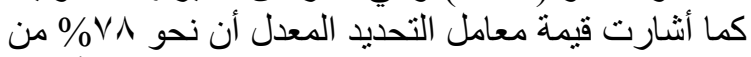

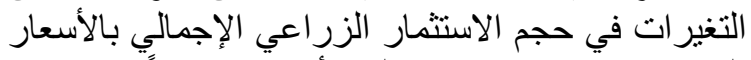

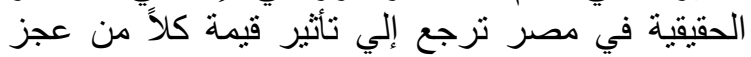

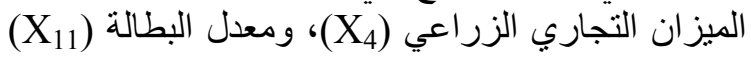

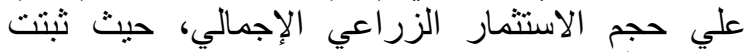

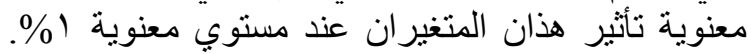

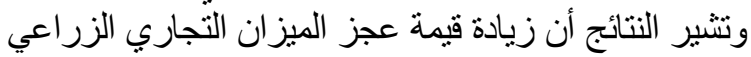

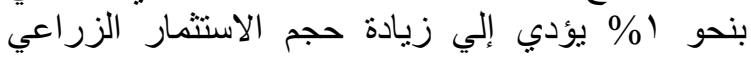

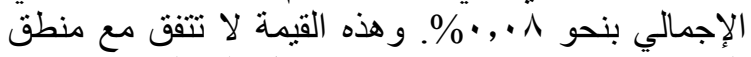

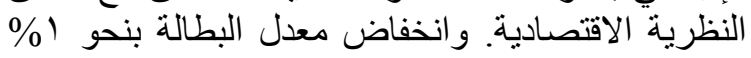

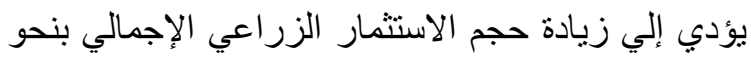
7 ، , • \% \% و هذه القيمة تتفق مع منطق النظرية الاقتصادية.

الصورة اللوغاريتمية المزدوجة

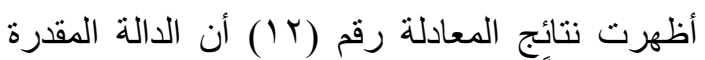

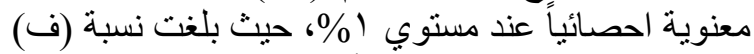

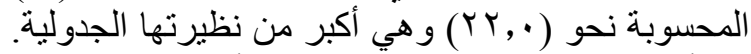

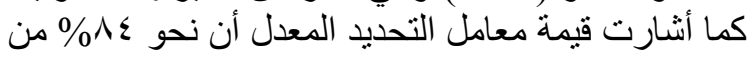

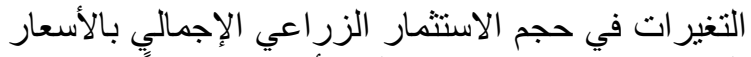

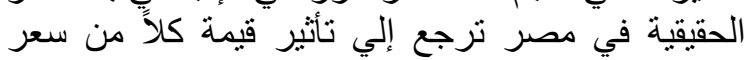

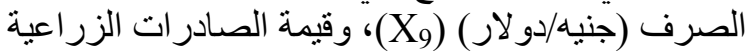

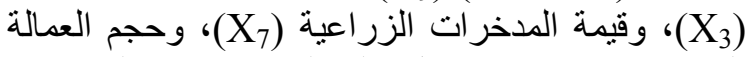

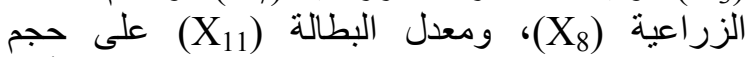
الاستثمار الزراعي الإجمالي، حيث ثبتث معند معنوية تأثير

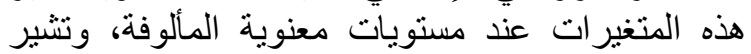

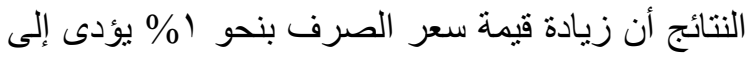

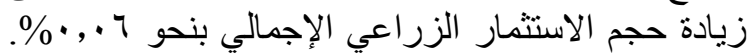

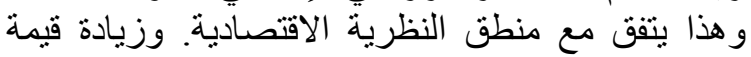


SINAI Journal of Applied Sciences (ISSN: 2314-6079), Vol. (8), Is. (1), Apr. 2019

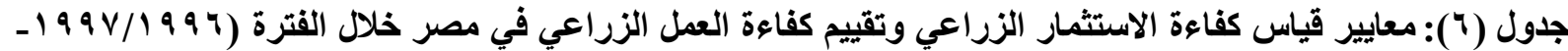
.$(Y \cdot 1 V / Y \cdot 17$

\begin{tabular}{|c|c|c|c|c|c|c|c|c|c|c|c|c|c|}
\hline \multirow{2}{*}{ 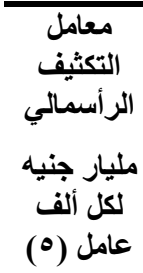 } & \multicolumn{3}{|c|}{ 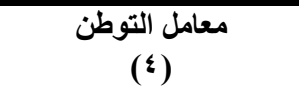 } & \multicolumn{3}{|c|}{ مضاعف الاستثمار } & \multicolumn{3}{|c|}{ 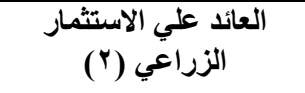 } & \multicolumn{3}{|c|}{ معدل الاستثمار الزراعي } & \multirow[t]{2}{*}{ السنوات } \\
\hline & جملة & خاص & عام & جملة & خاص & عام & جملة & خاص & عام عام & جملة & خاص & عام & \\
\hline $1,1$. & . & , §Y & $\bar{Y}$ & $0 \leqslant$ & $|Y Y, T|$ & $\bar{T}$ &,$\cdot V$ & $10, \pi r$ & $\bar{\lambda}$ & $\cdot, 1 Y$ & $\cdot, \cdot V$ & $r, \ldots$ & $1998 /$ \\
\hline $1, V$. & $\cdot, \vee_{0}$ &., 91 & $\varepsilon \varepsilon, q$. & $1, r V$ & $\Gamma, \Sigma ૫$ & $\cdot, \cdot 1$ & 0,09 & 11,94 & 。 & ᄉ & $\cdot, \cdot \wedge$ & $1, \times 0$ & $1991 / 199$ \\
\hline $1, \times 0$ & $\cdot, \times)$ & $\cdot, V T$ & $\leq 7, \cdot V$ & $1 r, 70$ & $\{, 7)$ & & 0,11 & $1 \cdot, \vee \wedge$ & 。 & $\cdot, I V$ & $\cdot, .9$ & $9,0$. & \\
\hline 1,77 & $\cdot, 70$ & $\cdot, T V$ & r^, ২০ & ऍ, & $৭, \vee \wedge$ & $\cdot, \cdot 1$ - & 7,0 . & $1 \cdot, v$. & $\cdot, \cdot v$ & $\cdot, 10$ & $\cdot, .9$ & 10,19 & $r$ \\
\hline $1,7 V$ & v & שד & . & 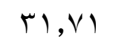 & $0, T V$ & $r_{-}$ & ,VY & אז, • & $\cdot, \cdot \wedge$ & $\cdot, 10$ & $\cdot, 1$. & $\mid \pi, 1 \leq$ & r \\
\hline $1,9 r$ & •, & $\cdot, 7)$ & rVI,I & $r, r V$ & 0,17 & . & $7, .9$ & $9, \wedge \wedge$ & $\cdot, \cdot 1$ & $\cdot, 17$ & $\cdot, 1$. & $v \varepsilon, \ldots$ & $\cdots 1$ \\
\hline $1, r_{0}$ & $\cdot, \leq \leqslant$ & . tro & דr, •rו & $1, \times 1-$ & r,. I- & $\cdot, \cdots$ & $9,9 \vee$ & $r ., .0$ & $\cdot, \cdot r$ & $\cdot, 1$. & $\cdot, .0$ & $T \varepsilon, \varepsilon$. & $r \ldots$ \\
\hline $1, \leqslant 0$ & $\cdot, Y V$ & שT, & $\| \wedge, r \wedge$ & $\vee, 97$ & rq,00 & $\cdot, \cdots$ & IT,VT & $r \leq, .0$ & $\cdot, \cdot 1$ & $\cdot, \cdot \wedge$ & $\cdot, \cdot \varepsilon$ & VI, r. & $r$. \\
\hline $1, \varepsilon r$ & • & $\cdot, r q$ & $1 \leqslant \pi, I V$ & $\mid \leq q, \vee 1$ & $\wedge \Gamma, \wedge \varepsilon_{-}$ &,,$\cdots$ & $1 ., 10$ & IV,V. & $\cdot, \cdot r$ & $\cdot, 1$. & $\cdot, .7$ & זr, & $r$. \\
\hline $1,0 Y$ & o & דוז, & $\Lambda V, .0$ & $1 \cdot, \leqslant 0$ & 7,01 &., 11 & $1 ., 1 \mathrm{~V}$ & $10,7$. & $\cdot, \cdot$ & $\cdot, 1$. &., .7 & - & $r$. \\
\hline $1, \varepsilon \leqslant$ & $\cdot$ & $\cdot, r Y$ & ז & $V Y_{1}, V Y_{-}$ & $101,0$. & $\cdot, \cdot T_{-}$ & Ir,Ar & $1 \wedge, 7 \leq$ & $\cdot, \cdot 1$ & $\wedge$ &., .0 & . & $r$ \\
\hline $1, \leqslant V$ & $\cdot, Y_{I}$ & $\cdot, 1 \leq$ & r & ,97 & qт,१५_ & $\cdot, \cdots$ & $1 \leq, \cdot 1$ & TI,T & $\cdot, \cdot 1$ & $\cdot, \cdot v$ &., .0 & $\leqslant r, 0$. & $r$. \\
\hline $1, r$. & $\cdot, 17$ &., $1 \mathrm{r}$ & ו & $\mid \wedge, \Sigma \Lambda_{-}$ & r.r., &,,$\cdots$ & 19,10 & r, & $\cdot, \cdot 1$ &., .0 & $\cdot, \cdot r$ & Irv,.. & $r \ldots q$ \\
\hline 1,17 & سו, &., .9 & $r \mid 9, r)$ & YIY,OA_ & I. r, . - & $\cdot, \cdot V$ & 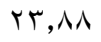 & $\{1,09$ & $\cdot, \cdot 1$ & $\cdot, \cdot \varepsilon$ & $\cdot, \cdot r$ & $97, \ldots$ & $r+1 \cdot / r \ldots q$ \\
\hline 1,11 & $\cdot, 11$ & $\cdot, \cdot 7$ & ו & Tr & $9 \leq, 17$ & $\cdot, \cdots$ & $r V, \wedge \varepsilon$ & or, \&l & $\cdot, \cdot 1$ & $\cdot, \cdot \varepsilon$ & $\cdot, \cdot r$ & سז, • 1 & $r$. \\
\hline$\cdot 9$. & $\cdot, I r$ &., .9 & $0 \leq, \wedge 1$ & $9 \leqslant$ & $1, \times 1$ & ـ & ro,17 & $7 q, \wedge \vee$ & $\cdot, .0$ & $\cdot, \cdot r$ & $\cdot,+1$ & $0 \leqslant$ & $r$. \\
\hline 1, ro & سז, &., 19 & 7. & 7,97 & $V, T V$ & $\cdot, \cdot V$ & ro, r r & ґА, ૫. & $\cdot$, & $\cdot, \cdot \leq$ & $\cdot, \cdot r$ & $19,7 V$ & $r$. \\
\hline $1, \times 1$ & $\cdot, \wedge r$ & $\cdot, \cdot 7$ & 71,99 & ov, . T_ & $q_{.}, \leqslant V_{-}$ & $\cdot, \cdot r$ & $r, .9$ & T,rT & $\cdot, \cdot \varepsilon$ & $\cdot, \leqslant \wedge$ & $\cdot, r$ & $r \varepsilon, \Sigma 1$ & $r$. \\
\hline$r, \cdot r$ & Tr, & ., r & $\{7, \vee \backslash$ & $1 \leqslant r, \vee q$ & ror,97 & , , r & $r \cdot, V V$ & سף, & $\cdot, \cdot \varepsilon$ &., .0 & r., & ru,.0 & $r$. \\
\hline$r, \varepsilon r$ & $\cdot$, ro & ., & זч, . & $1 \varepsilon, \cdot 1$ & $\mid r, Y \wedge$ & .,$r \varepsilon_{-}$ & 19,09 & rA, ro & $\cdot, .0$ &., .0 & $\cdot, \cdot \varepsilon$ & r), & 4.17 \\
\hline T,Y & ו & ., r & $\varepsilon \varepsilon, \cdot r$ & $1 \leq, Y \wedge$ & IT,VT & בז', & IN,Y & rr, & $\cdot, \cdot 7$ &., .0 & $\cdot, \cdot \varepsilon$ & $17,7 r$ & $r .1 V / r .17$ \\
\hline 1,09 & $\cdot, r q$ & . , rq & IVI, & rr, . q & $\mid r, .1$ & $\cdot, \cdot \mu_{-}$ & & $r \leqslant, r V$ & $\cdot, \cdot r$ &., 11 & $\cdot, \cdot v$ & $70, Y_{0}$ & المتوسط \\
\hline
\end{tabular}
الإشارة السالبة في مضاعف الاستثمار تشير إلي أن الاستثمار الزراعي في السنة الحالية أقل من الاستثمار الزراعي في السنة السابقة، أو أن الناتج

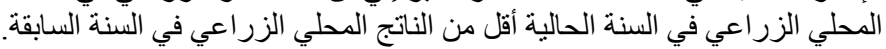

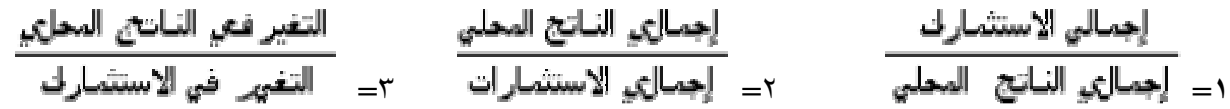

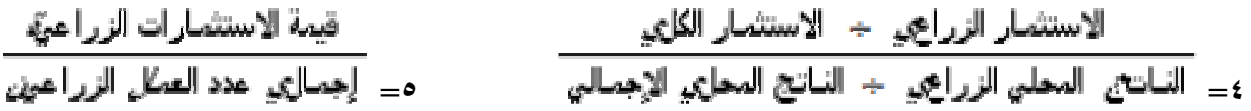


الزراعية في ضوء حجم الاستثمار ات اللازمة لإقامة

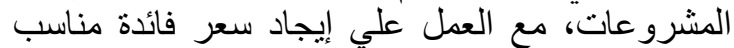

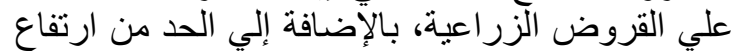

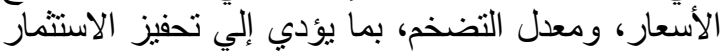

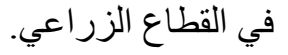

\section{المراجع}

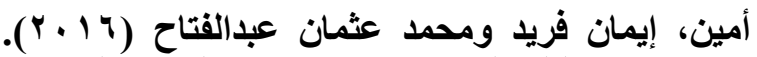
دراسة تحليلية لكفاءة الاستثمار في القطاع علئاع الزراعي

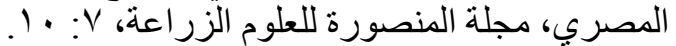

الجهاز المركزي للتعبئة العامة والاحصاء، نشرة التجارة الخارجية، أعداد متفرقة.

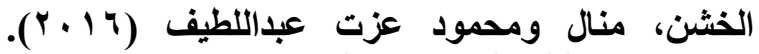
دراسة تحليلية للاستثمار الزرواعي في مصر، اعلى مجلة

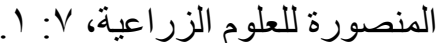

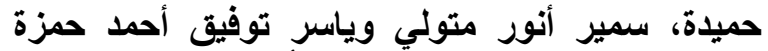

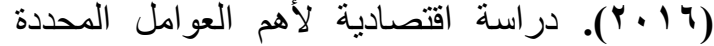

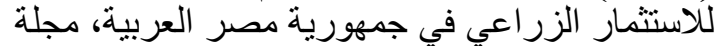

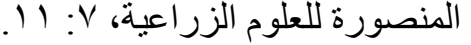

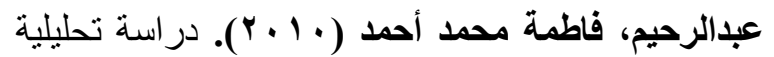

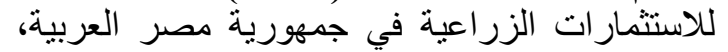
رسالة دكتور اه، جامعة قتاة السويس، كلية الزية الزراعة، الزاعة قسم الاقتصاد و الإرشاد و المجتمع الريفي.

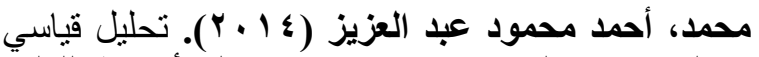

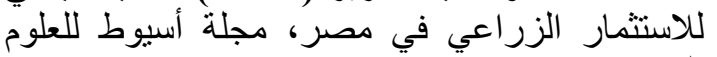

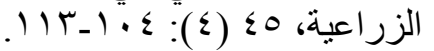

موقــع وزارة التخطيط والمتابعة والاصلاح الإداري. www.mop.gov.eg

موقع وزارة التنمية الاقتصادية علي شبكة المطومات

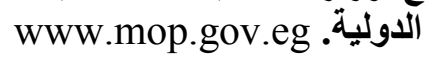

وزارة التنمية الاقتصادية، النشرات السنوية لخطط التنمية

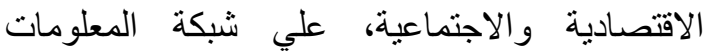

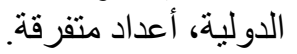

David, L.D. (2012). Agricultural Production Economics, Bibliography: p, Library of Congress Cataloging in Publication Data, $2^{\text {nd }}$ Ed.

Mahmoud, F.H. and H. Yokogawa (2000).

Agricltural investmentin Egypt and gapan (1978-1998) Acomparative study. Fac. Agr, Kyushu Univ., Japan.

Mary, A. and K. Wakelin (2003). Investment Liberalization and international Trade, J. Int. Econ., 61.

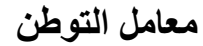

تؤكد النتائج إلي أن معامل التوطن لقطاع الزراعة

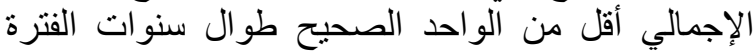

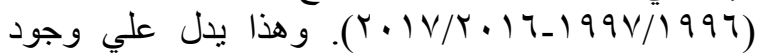

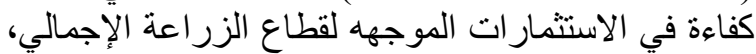

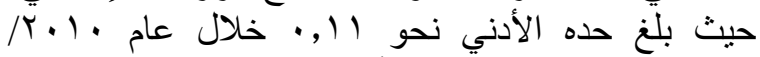

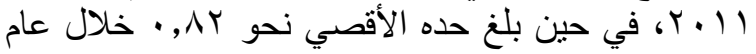

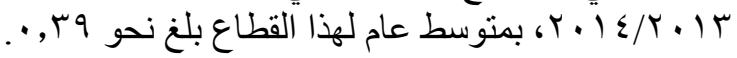
معامل التكثيف الرأسمالي (معامل التوظيف)

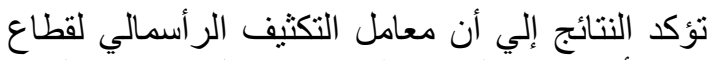

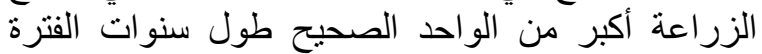

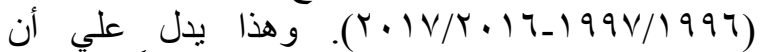
النشاط الاقتصادي في هذا القطاع يعتبر مكثفاً لاستخدام

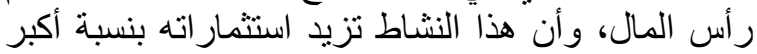

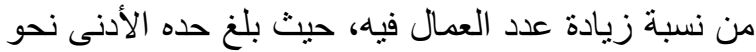

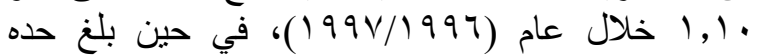

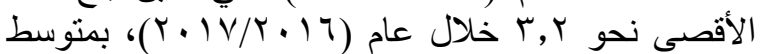

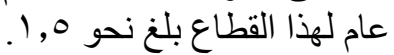
أهم التوصيات التي توصل إليها البحث

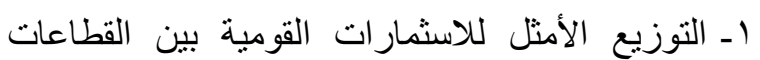

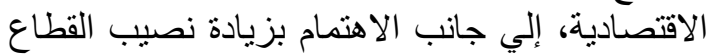

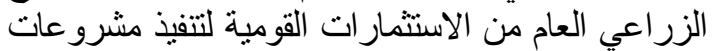

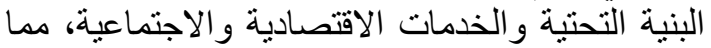
بساهم في خفض التكلفة الاستثمارية، ومن ثم زيادة الإنية الاستثمار ات الزر اعية.

Yـ تحسين بيئية الاستثمار في مصر من خلال: ـ تطوير أوضاع التسويق المحلي والعالمي والتصنيع

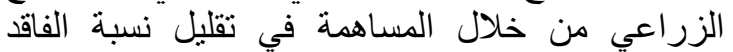

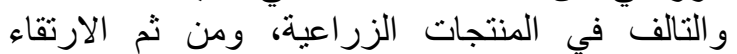

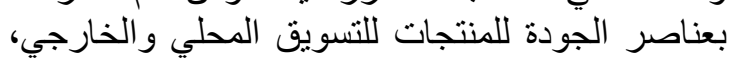

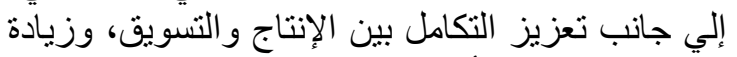
ربط المز ارعين بالأسواق و المتغير ات السئ والسية.

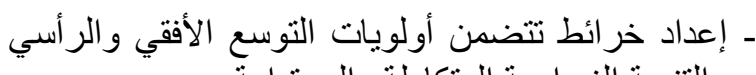
و التنمية الزر اعية المتكاملة و المستدامة.

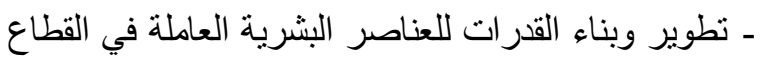

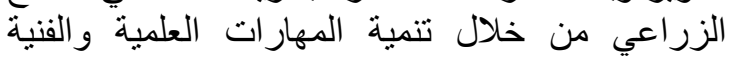

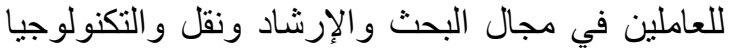

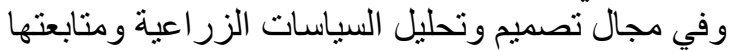

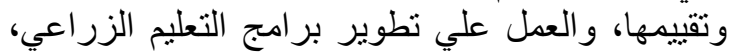

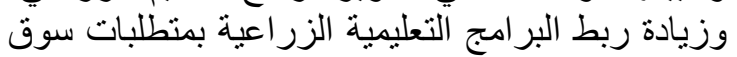
العمل و المستجدات الفنية و التطبيقية.

ـ تقديم تيسير ات للمنتفعين من الأراضي المستصلحة مثل الماتل

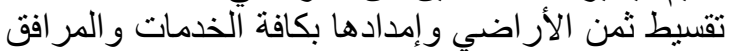
اللازمة، إلي جانب تحديد سعر الفائدة علي القروض 


\title{
AN ECONOMIC STUDY OF THE EFFICIENCY AND DETERMINANTS OF AGRICULTURAL INVESTMENT IN EGYPT
}

\author{
Wesam E.M.E. El-Ashram, R.I.M. Radwan, Soad A. Ibrahim and R.M. Hefny \\ Dept. Econ. and Rural Devlop., Fac. Environ. Agric. Sci., Arish Univ., Egypt.
}

\begin{abstract}
The agricultural sector had considered one of the main pillars on which the Egyptian economic structure depends. The average value of agricultural gross domestic product reached about LE 106.0 billion, representing about $9.7 \%$ of the average value of the national GDP which reached about LE 1091.6 billion during the period (1996/1997-2016/2017). Agriculture sector employs a large part of labor, which is about $28.6 \%$ of the total number of workers in economic activities, the average volume of national investments directed at agricultural sector reached about LE 9.1 billion, representing $8.5 \%$ of the average national investments, which amounted to LE 106.0 billion. The research problem is the decline in the share of the agricultural sector of the national investments, which decreased the relative importance of the agricultural investments volume in the total national investment from about $13.3 \%$ in (1996/1997), to about $9.4 \%$ in (2016/2017). The research aims to study agricultural investment efficiency and its determinants in Egypt, the research was based on descriptive analysis and quantitative analysis to characterize the economic variables, as well as the use of statistical analysis tools. With respect to the most important determinants affecting agricultural investment by real prices are the exchange rate, the unemployment rate, the interest rate of the banking system, the value of agricultural wages, the value of agricultural exports, and the value of agricultural savings. But in the semi-logarithmic form, the results confirmed that the value of the agricultural trade balance is increasing by $1 \%$ would increase agricultural investment volume of about $0.08 \%$. The unemployment rate is declining by $1 \%$ leads to increase agricultural investment volume about $0.06 \%$. As for the double logarithmic form, the value of the exchange rate, the value of agricultural savings and the volume of agricultural employment are increasing by about $1 \%$ would increase agricultural investment volume by about $0.06 \%, 0.75 \%, 0.24 \%$, respectively. The value of exports and the unemployment rate are decreasing by about $1 \%$ would increase agricultural investment volume about $0.67 \%, 0.04 \%$ respectively. The elasticity of agricultural investment was about 0.34 . With respect to the assessment of the efficiency of agricultural investments, the results confirmed that the rate of investment, return on investment, the investment multiplier, the coefficient of endemism, and the coefficient of capital intensive have reached about 0.11 , $14.3,22.0,0.3,1.5$, respectively. The study recommended the necessity of optimizing the distribution of national investments among the economic sectors, In addition to increasing the share of the national agricultural sector in the implementation of infrastructure projects economic and social services, the cost of investment is decreasing and agricultural investments are increasing.
\end{abstract}

Key words: Economic, efficiency, determinants of investment, investment efficiency. 
(ملحق)

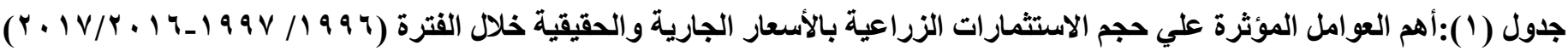

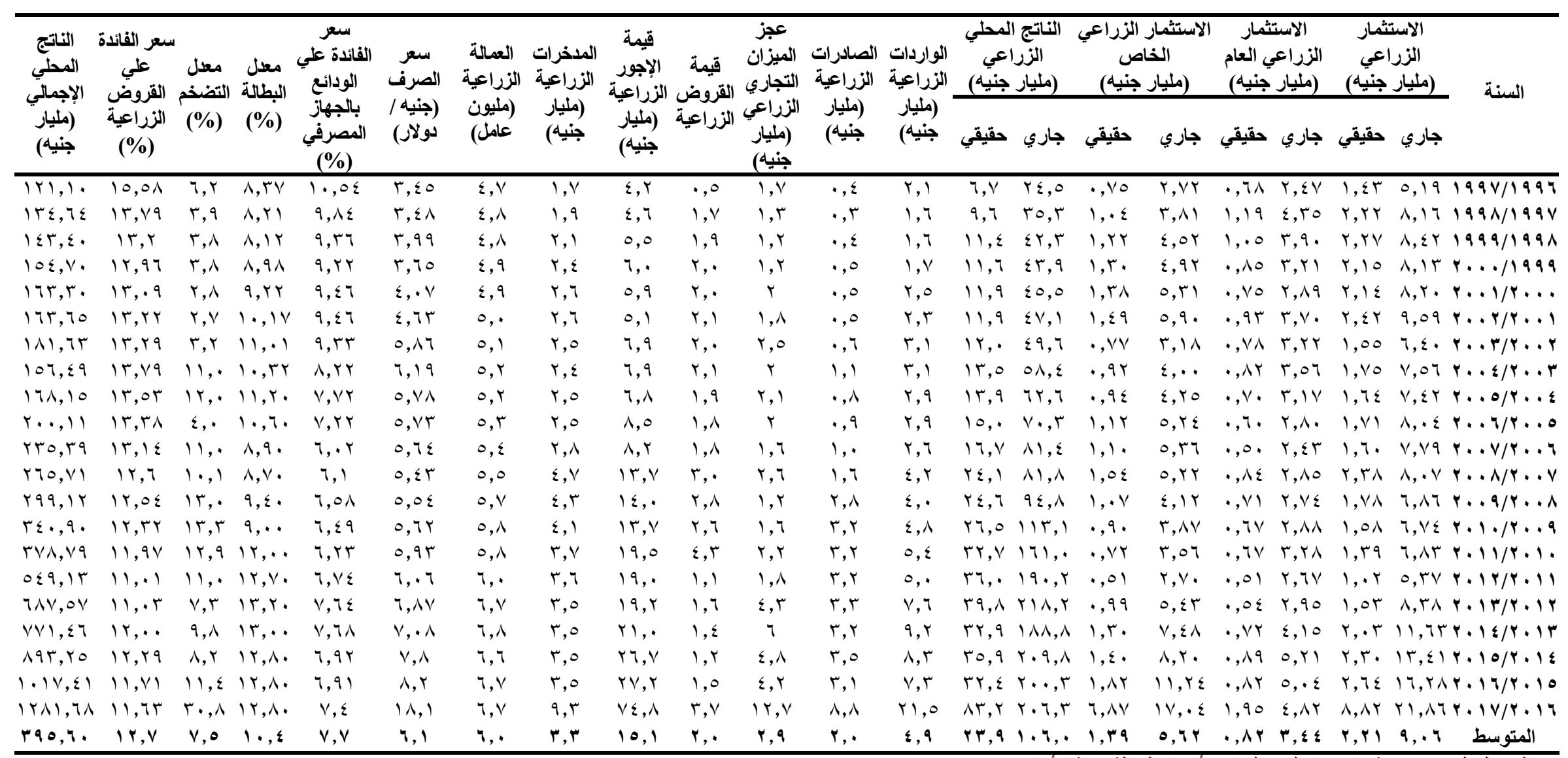

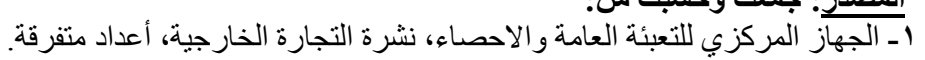

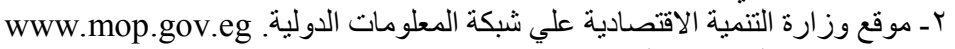
سـ موقع وزارة التخطيط و المتابعة والآصلاح الإداري. 